\title{
Adaptive Iterative Learning Control for Nonlinear Uncertain Systems with Both State and Input Constraints
}

\author{
Qiongxia Yua ${ }^{\mathrm{a}}$, Zhongsheng Hou ${ }^{\mathrm{a}, *}$, Ronghu Chi ${ }^{\mathrm{b}}$ \\ ${ }^{a}$ Advanced Control Systems Lab, School of Electronic and Information Engineering, Beijing Jiaotong \\ University, Beijing 100044, PR China \\ ${ }^{b}$ School of Automation and Electronic Engineering, Qingdao University of Science and Technology, \\ Qingdao 266042,PR China
}

\begin{abstract}
This work proposes a new adaptive iterative learning control (AILC) scheme for nonlinear systems with both state and input constraints, where the time-varying parametric uncertainties, external disturbances, and random initial errors are also considered together. The proposed AILC consists of a learning control law and two fully projected parameter learning laws. By incorporating a barrier composite energy function into the learning control law and using a projection mechanism for the parameter learning laws, the proposed AILC can flexibly and actively manipulate the states and inputs of the system into their pre-specified and constrained ranges, respectively. It is theoretically shown that the asymptotic and pointwise convergence properties are guaranteed without violating any state and input constraints. The validity of the proposed AILC scheme is further verified with a practical train operation system.
\end{abstract}

Keywords: adaptive iterative learning control, state constraints, input constraints, random initial conditions, external disturbances, train operation system

\section{Introduction}

Many real systems execute repeatable tasks over a finite time interval, such as robot manipulators [1], linear motor systems [2], freeway traffic [3], batch reactor [4], and train operation systems [5, 6]. However, traditional approaches [7-10] do not consider the repeatability of the practical processes and cannot learn from previous operations. Comparatively, learning control $[11,12]$ can learn from experience to improve control performance. In general, there are two main types of learning control-repetitive control (RC) and iterative learning control (ILC). Repetitive control (RC) operates on the infinite time domain and is a typical method for tracking periodic trajectories or rejecting periodic /periodic-like exogenous disturbances [13-15], such as the applications in the

* Corresponding author.

E-mail addresses: qiongxiayu@hotmail.com, zhshhou@bjtu.edu.cn,ronghu_chi@hotmail.com

(C) 2016. This manuscript version is made available under the Elsevier user license http://www.elsevier.com/open-access/userlicense/1.0/ 
hydraulic and electrical motor actuation [16, 17]. Compared with RC, iterative learning control (ILC) is operating in a finite time interval, and is a well-known method for tracking repeatable control tasks over a finite time interval in iteration domain. The objective of ILC is to improve the control performance of the current execution (iteration) by using tracking performance information and the control efforts of previous executions (iterations). Since it was proposed by Arimoto [18], ILC has achieved significant developments [1-6, 19-24].

Note that many real systems are subjected to input and state constraints due to physical limitations of the actuators or the safety requirements. For instance, unmanned aerial vehicle (UAV) flight control systems $[25,26]$ are subjected to input constraint owing to the limited size of the actuators. Besides input constraint, state constraint is always encountered simultaneously. For a train operation system, it is constrained not only by the input force because of its mechanical features, but also by its operation speed for reason of high safety requirement[6].

For the repetitive systems with both input and output/state constraints, some works on model predictive ILC [4, 27-30] and optimal ILC [31-33] have been proposed recently. Although these approaches [30-32] can incorporate constraint handling by minimizing a cost function subjected to input and output constraints, a main limitation is that a perfect linear model should be known exactly for the controller design and analysis. Otherwise, the satisfactory control performance is no longer guaranteed.

However, many real plants are nonlinear with various uncertainties, so that the accurate physical system model is hard to obtain. Taking the high-speed train operation system as an example, although it repetitively runs according to its fixed operation timetable every day, the uncertainties related to the aerodynamics resistance on the track due to different climate conditions and the load disturbances due to operationdependent passenger numbers, are always encountered.

It is well known that adaptive ILC approach has many advantages in dealing with system uncertainties by making full use of the information of system structure and the repeatable operation pattern. In recent years, some novel adaptive ILC schemes $[1,19,34-41]$ have been developed by introducing adaptive control mechanism into iteration domain. However, only few works $[40,41]$ about adaptive ILC have been done for system with state or input constraints. In [40], the authors discussed the state constraints by utilizing barrier composite energy function (BCEF). In [41], a reference governor (RG) is introduced to deal with input saturation. It is worth noting that the existing results $[40,41]$ deal with input constraints and state constraints separately.

Another issue in the adaptive ILC for constrained systems is that initial states in each iteration should be the same [41] or satisfy alignment condition [40], and the external disturbances should be repetitive in all iterations. Obviously, identical initial condition and iteration-independent external disturbances are strict assumptions in practice due to the real-time uncertainties and measurement noises. The alignment condition means that the terminal state of the previous operation becomes the initial state of the current operation. In fact, this initial condition is not easily satisfied in many practical applications. For example, the terminal position of a high-speed train in the previous operation cannot be regarded as the initial position of the current operation because the high-speed train runs repetitively from the same departure station to the terminal station according to the operation schedule. In the field of adaptive IL- 
$\mathrm{C}$, although some works have been reported to address iteration-varying initial errors $[35,36,39]$ and iteration-dependent disturbances [37-39], these results do not consider any constraints on state or input.

In summary, up to now, little work about adaptive ILC has been reported to address the systems under both state and input constraints, together with system uncertainties. Therefore in this work, a novel adaptive iterative learning control (AILC) scheme is proposed. It is the first time that both state and input constraints, together with timevarying parametric uncertainties, time-iteration-varying external disturbances and random initial conditions, are all addressed for nonlinear systems. The distinct features of the proposed AILC are as follows:

(1) Compared with the adaptive control in time domain, the proposed AILC can learn from repetitive operations such that it is able to address time-varying parametric uncertainties which can be considered as constant ones along the iteration axis.

(2) In comparison with the existing adaptive ILC, this new AILC not only can deal with time-varying parameters, random initial errors, and iteration-dependent external disturbances simultaneously, but also can guarantee both state and input constraints.

(3) In contrast to the existing ILC with constraints that the signals are passively limited by a saturator, this new AILC directly considers state and input constraints in the controller design, and both state and input signals can be auto-regulated into the constrained ranges.

Moreover, a train operation system is considered in the simulation study as a typical representative of nonlinear uncertain systems with both state and input constraints. And the effectiveness and applicability of the proposed method are verified further.

The rest of this work is as follows. Section 2 is the problem formulation. The proposed adaptive ILC scheme is developed in Section 3 with rigorous convergence analysis. Section 4 validates the applicability of the proposed AILC in the train operation system. Conclusions are given in Section 5.

In the following discussion, the time index $t$ is dropped when no confusion arises.

\section{Problem formulation}

The following nonlinear continuous-time system is considered [21, 39]:

$$
\begin{gathered}
\dot{x}_{j, k}(t)=x_{j+1, k}(t), \quad j=1,2, \ldots, n-1 \\
\dot{x}_{n, k}(t)=\boldsymbol{\theta}^{T}(t) \boldsymbol{\xi}\left(\mathbf{x}_{k}(t)\right)+u_{k}(t)+d_{k}(t)
\end{gathered}
$$

where the subscript $k \in \mathbf{Z}_{+}$represents the iteration index; $t \in[0, T]$ denotes the time index; $\mathbf{x}_{k}(t)=\left[x_{1, k}(t), x_{2, k}(t), \ldots, x_{n, k}(t)\right]^{T}$ and $u_{k}(t)$ are accessible states and input, respectively; $\boldsymbol{\theta}(t)=\left[\theta_{1}(t), \ldots, \theta_{m}(t)\right]^{T} \in \mathfrak{R}^{m \times 1}$ is an unknown and bounded parameter vector with respect to time $t . \boldsymbol{\xi}\left(\mathbf{x}_{k}(t)\right)=\left[\boldsymbol{\xi}_{1}\left(\mathbf{x}_{k}(t)\right), \xi_{2}\left(\boldsymbol{x}_{k}(t)\right), \ldots, \boldsymbol{\xi}_{m}\left(\mathbf{x}_{k}(t)\right]^{T} \in \mathfrak{R}^{m \times 1}\right.$ denotes a known vector function with respect to the states; $d_{k}(t)$ is an unknown and bounded external disturbance.

The desired state vector $\mathbf{p}_{k}(t)=\left[p_{1, k}(t), p_{2, k}(t), \ldots, p_{n, k}(t)\right]^{T}$ is assumed to be bounded by

$$
\left|p_{j, k}(t)\right| \leq \eta_{j}, \quad j=1,2, \ldots, n
$$


where $\eta_{1}, \eta_{2}, \ldots, \eta_{n}$ are known positive constants, and is generated by [21]

$$
\begin{aligned}
& \dot{p}_{j, k}(t)=p_{j+1, k}(t), \quad j=1,2, \ldots, n-1 \\
& \dot{p}_{n, k}(t)=h\left(\mathbf{p}_{k}(t), t\right)
\end{aligned}
$$

where $h\left(\mathbf{p}_{k}(t), t\right)$ is a known and bounded vector function.

Since $d_{k}(t)$ and $h\left(\mathbf{p}_{k}(t), t\right)$ are bounded, it is reasonable to assume that there exists an unknown positive constant $\rho$ such that

$$
\left|h\left(\mathbf{p}_{k}(t), t\right)-d_{k}(t)\right| \leq \rho
$$

In this work, system (1) is subjected to the following two constraints:

(1) Input constraint:

$$
\left|u_{k}(t)\right| \leq u_{\max }
$$

where $u_{\max }$ denotes the maximum limit of the control input $u_{k}(t)$.

(2) State constraint:

$$
\left|x_{j, k}(t)\right| \leq x_{j, \max }, \quad j=1,2, \ldots, n
$$

where $\mathbf{x}_{\max }=\left[x_{1, \max }, \ldots, x_{n, \max }\right]^{T}$ is the maximum limit of the state vector $\mathbf{x}_{k}(t)$.

Without violating the above input constraint (5) and state constraints (6), the control objective of this work is to find the control input $u_{k}(t)$ that drives the state $\mathbf{x}_{k}(t)$ to track the desired $\mathbf{p}_{k}(t)$, by considering random initial state errors, parametric uncertainties and unknown external disturbances simultaneously.

Denote the state error vector $\mathbf{e}_{k}(t)=\left[e_{1, k}(t), e_{2, k}(t), \ldots, e_{n, k}(t)\right]^{T}=\mathbf{p}_{k}(t)-\mathbf{x}_{k}(t)$, where $e_{j, k}(t)=p_{j, k}(t)-x_{j, k}(t), j=1,2, \ldots, n$. According to (1) and (3), It is straightforward to derive that $\left[e_{1, k}(t), e_{2, k}(t), e_{3, k}(t), \ldots\right]^{T}=\left[e_{1, k}(t), \dot{e}_{1, k}(t), \ddot{e}_{1, k}(t), \ldots\right]^{T} \triangleq\left[e_{1, k}^{(0)}\right.$ $\left.(t), e_{1, k}^{(1)}(t), e_{1, k}^{(2)}(t), \ldots\right]^{T}$.

Assumption (A1). The initial state error $\mathbf{e}_{k}(0)=\left[e_{1, k}(0), e_{2, k}(0), \ldots, e_{n, k}(0)\right]^{T}$ is random and bounded, i.e., for $\forall k \in \mathbf{Z}_{+}$

$$
\left|e_{j, k}(0)\right| \leq \delta_{j}, \quad j=1,2, \ldots, n
$$

where $\delta_{1}, \delta_{2}, \ldots, \delta_{n}$ are the upper bounds of the initial state errors $e_{1, k}(0), e_{2, k}(0), \ldots$, $e_{n, k}(0)$ respectively and they are known positive constants.

Remark 1. In the traditional ILC design [21, 34, 37], the initial states of the system should be identical with the desired ones at each iteration, namely $x_{j, k}(0)=p_{j, k}(0), j=$ $1,2, \ldots, n$. Consequently, the initial state error $e_{j, k}(0)$ should strictly be zero at each iteration. By contrast, Assumption A1 relaxes this strict restriction. In Assumption A1, the initial state errors at each iteration can be randomly varying as long as they are bounded. 


\section{Constrained Adaptive ILC Design}

Define an extended error as

$$
\begin{aligned}
s_{k}(t) & =\lambda_{1} e_{1, k}(t)+\lambda_{2} e_{2, k}(t)+\cdots+\lambda_{n-1} e_{n-1, k}(t)+e_{n, k}(t) \\
& =\lambda^{T} \mathbf{e}_{k}(t)
\end{aligned}
$$

where $\lambda=\left[\lambda_{1}, \lambda_{2}, \ldots, \lambda_{n-1}, 1\right]^{T}$. Note that $s_{k}(t)$ can be rewritten as $s_{k}(t)=\left(\left(d^{n-1}\right.\right.$ $\left.\left./ d t^{n-1}\right)+\lambda_{n-1}\left(d^{n-2} / d t^{n-2}\right)+\cdots+\lambda_{1}\right) e_{1, k}(t) \triangleq \Delta(d) e_{1, k}(t) . \Delta(d)$ denotes the Hurwitz polynomial with $\lambda_{1}, \lambda_{2}, \ldots, \lambda_{n-1}$ being the coefficients.

According to Assumption A1, one can find a constant $\delta$ such that $s_{k}(0)$ satisfies

$$
\begin{aligned}
\left|s_{k}(0)\right| & =\left|\lambda_{1} e_{1, k}(0)+\cdots+\lambda_{n-1} e_{n-1, k}(0)+e_{n, k}(0)\right| \\
& \leq \lambda_{1} \delta_{1}+\cdots+\lambda_{n-1} \delta_{n-1}+\delta_{n} \leq \delta
\end{aligned}
$$

To overcome the random initial state errors, an auxiliary error $s_{k}^{\phi}(t)$ is introduced as follows[39]:

$$
s_{k}^{\phi}(t)=s_{k}(t)-\phi(t) \operatorname{sat}\left(s_{k}(t) / \phi(t)\right)
$$

where the operator sat $(\cdot)$ denotes the saturation function:

$$
\operatorname{sat}\left(\frac{s_{k}(t)}{\phi(t)}\right)=\left\{\begin{array}{ccc}
1 & \text { if } & s_{k}(t)>\phi(t) \\
s_{k}(t) / \phi(t) & \text { if } & \left|s_{k}(t)\right| \leq \phi(t) \\
-1 & \text { if } & s_{k}(t)<-\phi(t)
\end{array}\right.
$$

and $\phi(t)$ is the width of the boundary layer, satisfying the following separable differential equation:

$$
\dot{\phi}(t)+\beta \phi(t)=0
$$

Note that $\phi(t)$ is a decreasing function along the time axis that specifies the nonlinear boundary function with the initial condition $\phi(0)=\delta$ and $\beta>0$. $\delta$ is denoted by equation (9) to cope with the state bias in the initial stage of each iteration. It can be derived directly that $0<\delta \exp (-\beta T) \leq \phi(t) \leq \delta$ for all $t \in[0, T]$. In addition, it can be seen that the larger $\beta$ is, the smaller $\phi(t)$ will be. If $\lim _{k \rightarrow \infty} s_{k}^{\phi}(t)=0, \forall t \in[0, T]$, then $\lim _{k \rightarrow \infty}\left|s_{k}(t)\right| \leq \phi(t)$ in terms of equation (10).

For the subsequent analysis of the convergence property, three prominent properties of $s_{k}^{\phi}(t)$ are shown as follows:

$$
\begin{aligned}
& s_{k}^{\phi}(0)=0 \\
& s_{k}^{\phi}(t) s a t\left(\frac{s_{k}(t)}{\phi(t)}\right)= \\
& =\mid \begin{array}{ll}
s_{k}(t)-\phi(t), & \text { if } s_{k}(t)>\phi(t) \\
0, & \text { if }\left|s_{k}(t)\right| \leq \phi(t) \\
-\left(s_{k}(t)+\phi(t)\right), & \text { if } s_{k}(t)<-\phi(t)
\end{array} \\
& =\left|s_{k}^{\phi}(t)\right|
\end{aligned}
$$




$$
\text { if } \quad\left|s_{k}(t)\right|>\phi(t) \quad \dot{s}_{k}^{\phi}(t)=\dot{s}_{k}(t)-\operatorname{sgn}\left(s_{k}^{\phi}(t)\right) \dot{\phi}(t)
$$

Then, the proposed AILC at the $k$-th iteration is constructed as:

$$
\begin{aligned}
u_{k}(t)= & \beta s_{k}(t)+\left|\overline{\boldsymbol{\lambda}}^{T} \mathbf{e}_{k}(t)\right| \operatorname{sat}\left(\frac{s_{k}(t)}{\phi(t)}\right) \\
& -\hat{\boldsymbol{\theta}}_{k}^{T}(t) \boldsymbol{\xi}\left(\mathbf{x}_{k}(t)\right)+\hat{\boldsymbol{\rho}}_{k}(t) \operatorname{sat}\left(\frac{s_{k}(t)}{\phi(t)}\right)
\end{aligned}
$$

where $\beta$ is defined in equation (12); $\bar{\lambda}=\left[0, \lambda_{1}, \lambda_{2}, \ldots, \lambda_{n-1}\right]^{T} ; \hat{\boldsymbol{\theta}}_{k}(t)=\left[\hat{\boldsymbol{\theta}}_{1, k}(t), \ldots\right.$, $\left.\hat{\boldsymbol{\theta}}_{m, k}(t)\right]^{T}$ is to estimate $\boldsymbol{\theta}(t) ; \hat{\rho}_{k}(t)$ is to learn the upper bound of external disturbance $\rho$, which is defined in inequality (4).

The fully projected parameter learning laws for $\hat{\boldsymbol{\theta}}_{k}(t)$ and $\hat{\rho}_{k}(t)$ are constructed as

$$
\begin{aligned}
\hat{\boldsymbol{\theta}}_{k}^{*}(t) & =\hat{\boldsymbol{\theta}}_{k-1}(t)-\boldsymbol{\Lambda} \boldsymbol{\xi}\left(\mathbf{x}_{k}(t)\right) \alpha_{k}(t) \\
\hat{\rho}_{k}^{*}(t) & =\hat{\rho}_{k-1}(t)+v\left|\alpha_{k}(t)\right| \\
\hat{\boldsymbol{\theta}}_{k}(t) & =\operatorname{proj}_{\theta}\left(\hat{\boldsymbol{\theta}}_{k}^{*}(t)\right), \quad \hat{\boldsymbol{\theta}}_{-1}(t)=0 \\
\hat{\rho}_{k}(t) & =\operatorname{proj}_{\rho}\left(\hat{\rho}_{k}^{*}(t)\right), \quad \hat{\rho}_{-1}(t)=0
\end{aligned}
$$

where $\alpha_{k}(t) \triangleq \frac{s_{k}^{\phi}(t)}{=} \frac{\cos ^{2}\left(\pi s_{k}^{\phi 2}(t) / 2 k_{v}^{2}\right)}{\cos ^{2}}, k_{v}>0$ is the constraint on $s_{k}^{\phi}(t) . \quad \mathbf{\Lambda} \in \mathfrak{R}^{m \times m}$ is the parametric learning gain matrix which is symmetric positive definite. $v$ is a positive learning gain. The unknown parameters $\boldsymbol{\theta}(t)$ and $\rho$ can be estimated by $\hat{\boldsymbol{\theta}}_{k}^{*}(t)$ and $\hat{\rho}_{k}^{*}(t)$ in equation (17) and (18) respectively. In order to keep these estimations from diverging and retain them within feasible bounds, the projection mechanisms $\operatorname{proj}_{\theta}$ and $\operatorname{proj}_{\rho}$ are applied to $\hat{\boldsymbol{\theta}}_{k}^{*}(t)$ and $\hat{\rho}_{k}^{*}(t)$ in (19) and (20) such that

$$
\begin{array}{r}
\operatorname{proj}_{\theta}\left(\hat{\boldsymbol{\theta}}_{k}^{*}(t)\right)=\left\{\operatorname{proj}_{\theta}\left(\hat{\boldsymbol{\theta}}_{j, k}^{*}(t)\right)\right\} \quad(j=1,2, \ldots, m) \\
\operatorname{proj}_{\theta}\left(\hat{\theta}_{j, k}^{*}(t)\right)= \begin{cases}\theta_{j, \min } & \hat{\boldsymbol{\theta}}_{j, k}^{*}(t)<\theta_{j, \min } \\
\hat{\boldsymbol{\theta}}_{j, k}^{*}(t) & \theta_{j, \min } \leq \hat{\theta}_{j, k}^{*}(t) \leq \theta_{j, \max } \\
\theta_{j, \max } & \hat{\boldsymbol{\theta}}_{j, k}^{*}(t)>\theta_{j, \max }\end{cases}
\end{array}
$$

and

$$
\operatorname{proj}_{\rho}\left(\hat{\rho}_{k}^{*}(t)\right)= \begin{cases}\rho_{\min } & \hat{\rho}_{k}^{*}(t)<\rho_{\min } \\ \hat{\rho}_{k}^{*}(t) & \rho_{\min } \leq \hat{\rho}_{k}^{*}(t) \leq \rho_{\max } \\ \rho_{\max } & \hat{\rho}_{k}^{*}(t)>\rho_{\max }\end{cases}
$$

where $\boldsymbol{\theta}_{\min }=\left[\theta_{1, \min }, \theta_{2, \min }, \ldots, \theta_{m, \text { min }}\right]^{T}$ and $\boldsymbol{\theta}_{\max }=\left[\theta_{1, \max }, \theta_{2, \text { max }}, \ldots, \theta_{m, \max }\right]^{T}$ are the lower and upper bounds for $\hat{\boldsymbol{\theta}}_{k}(t) . \rho_{\min }$ and $\rho_{\max }$ are the lower and upper bounds for $\hat{\rho}_{k}(t)$. Assume that $\theta_{j}(t)=\operatorname{proj}_{\theta}\left(\theta_{j}(t)\right) j=1,2, \ldots, m$, and $\rho=\operatorname{proj}_{\rho}(\rho)$, i.e., we assume that $\boldsymbol{\theta}(t)$ lies within the range between $\boldsymbol{\theta}_{\min }$ and $\boldsymbol{\theta}_{\max }$, and $\rho$ lies within the range between $\rho_{\min }$ and $\rho_{\max }$.

Remark 2. The computational complexity of the proposed AILC (16)-(20) measured by the number of floating point operations (FLOPs)[42] is analyzed here. Define the 
sample time as $t_{s}$, then the total FLOPs for (16) at each sample time is $2 m^{2}+3 m+$ $2 n+6$, where $m$ and $n$ are the dimension of the unknown parameter and the system state. Since the operation time $t \in[0, T]$, the FLOPs over the whole time interval for one iteration can be given by FLOPs $=\left(2 m^{2}+3 m+2 n+6\right) \frac{T}{t_{s}}$.

Before proceeding to the convergence property, the following lemma regarding the projection mechanism is introduced.

Lemma 1. For $\forall \boldsymbol{\theta}(t) \in\left[\boldsymbol{\theta}_{\min }, \boldsymbol{\theta}_{\max }\right]^{T}$ and $\rho \in\left\{\rho_{\min }, \rho_{\max }\right\}$, we have[35]

$$
\left[\boldsymbol{\theta}(t)-\operatorname{proj}_{\theta}\left(\hat{\boldsymbol{\theta}}_{k}^{*}(t)\right)\right]^{T} \boldsymbol{\Lambda}^{-1}\left[\hat{\boldsymbol{\theta}}_{k}^{*}(t)-\operatorname{proj}_{\theta}\left(\hat{\boldsymbol{\theta}}_{k}^{*}(t)\right)\right] \leq 0
$$

and

$$
\left(\rho-\operatorname{proj}_{\rho}\left(\hat{\rho}_{k}^{*}(t)\right)\right)\left(\hat{\rho}_{k}^{*}(t)-\operatorname{proj}_{\rho}\left(\hat{\rho}_{k}^{*}(t)\right)\right) \leq 0
$$

The convergence of the proposed AILC is guaranteed by the following theorem.

Theorem 1. For the system (1) that meets Assumption A1, the learning control law (16) and the parameter learning laws (17)-(20) guarantee that

(T1) $s_{k}^{\phi}(t)$ approaches to zero asymptotically along the iteration axis for $\forall t \in[0, T]$, namely $\lim _{k \rightarrow \infty} s_{k}^{\phi}(t)=0$;

(T2) $\lim _{k \rightarrow \infty}\left|s_{k}(t)\right| \leq \phi(t) \leq \delta, \forall t \in[0, T]$;

(T3) the state errors $\mathbf{e}_{k}(t)=\left[e_{1, k}(t), e_{2, k}(t), \ldots, e_{n, k}(t)\right]^{T}=\left[e_{1, k}^{(0)}(t), e_{1, k}^{(1)}(t), \ldots, e_{1, k}^{(n-1)}\right.$ $(t)]^{T}$ converge to a tunable set asymptotically along the iteration axis for $\forall t \in$ $[0, T]$, namely $\lim _{k \rightarrow \infty}\left|e_{1, k}^{(i)}(t)\right| \leq\left[\frac{\delta}{c^{n-1-i}}+\sigma_{i}\right] 2^{i}, i=0,1, \ldots, n-1 . c>0$ is a constant defined in the following (50). $\sigma_{i} \triangleq \lim _{k \rightarrow \infty} \sum_{z=1}^{n-1-i} \frac{\left.\left[\left(\frac{d}{d t}+c\right)^{n-z-1}\left|e_{1, k}(t)\right|\right]\right|_{t=0}}{c^{n-z-i-1}}$ related to the initial state errors, and $\sum_{z=l}^{q}(\cdot)_{z} \triangleq 0$ for $l>q$.

(T4) $s_{k}^{\phi}(t), s_{k}(t), \mathbf{e}_{k}(t), \mathbf{x}_{k}(t)$, and $u_{k}(t)$ are all bounded for any time in all iterations; meanwhile, the input constraint (5) and the state constraints (6) can always be obeyed.

Proof. In order to facilitate the analysis of convergence property under constrained condition, we define a positive definite Barrier Composite Energy Function (BCEF) at the $k$-th iteration as

$$
\begin{aligned}
\bar{E}_{k}(t) & =\frac{k_{v}^{2}}{\pi} \tan \left(\frac{\pi s_{k}^{\phi 2}(t)}{2 k_{v}^{2}}\right)+\frac{1}{2} \int_{0}^{t} \tilde{\boldsymbol{\theta}}_{k}^{T}(\tau) \boldsymbol{\Lambda}^{-1} \tilde{\boldsymbol{\theta}}_{k}(\tau) d \tau+\frac{1}{2 v} \int_{0}^{t} \tilde{\rho}_{k}^{2}(\tau) d \tau \\
& \triangleq \bar{W}_{k}+\bar{N}_{k}+\bar{L}_{k}
\end{aligned}
$$

where $\bar{W}_{k} \triangleq \frac{k_{v}^{2}}{\pi} \tan \left(\frac{\pi s_{k}^{\phi 2}(t)}{2 k_{v}^{2}}\right), \tan (\cdot)$ is a tangent function; $\tilde{\boldsymbol{\theta}}_{k}(t)=\boldsymbol{\theta}(t)-\hat{\boldsymbol{\theta}}_{k}(t), \tilde{\rho}_{k}(t)=$ $\rho-\hat{\rho}_{k}(t) . \bar{N}_{k} \triangleq \frac{1}{2} \int_{0}^{t} \tilde{\boldsymbol{\theta}}_{k}^{T}(\tau) \boldsymbol{\Lambda}^{-1} \tilde{\boldsymbol{\theta}}_{k}(\tau) d \tau, \bar{L}_{k} \triangleq \frac{1}{2 v} \int_{0}^{t} \tilde{\rho}_{k}^{2}(\tau) d \tau$. 
It should be noted that the state and input constraints will be guaranteed by virtue of this defined BCEF (25), and the following proof of (T4) will demonstrate how these constraints can always be satisfied in detail. A further explanation will be given in the subsequent Remark 3.

Now, (T1)-(T4) in Theorem 1 are proved respectively.

(T1) The proof consists of two parts. Part 1 derives the negative definite difference of $\bar{E}_{k}(t)$. Part 2 derives the boundedness of BCEF $\bar{E}_{k}(t)$ and proves the asymptotic and pointwise convergence properties of $s_{k}^{\phi}(t)$.

Note that according to equations (10) and (11), when $\left|s_{k}(t)\right| \leq \phi(t)$, it is straightforward to derive that $s_{k}^{\phi}(t)=0$. Consequently, the subsequent proof is in the case of $\left|s_{k}(t)\right|>\phi(t)$.

Part 1 of the proof of (T1). The negative definite difference of $\bar{E}_{k}$

Make a difference between $\bar{E}_{k}$ and $\bar{E}_{k-1}$,

$$
\begin{aligned}
\Delta \bar{E}_{k} & =\bar{E}_{k}-\bar{E}_{k-1} \\
& =\bar{W}_{k}-\bar{W}_{k-1}+\bar{N}_{k}-\bar{N}_{k-1}+\bar{L}_{k}-\bar{L}_{k-1}
\end{aligned}
$$

According to (13) and (15), the first term on the right side of equation (26), namely $\bar{W}_{k}$, becomes

$$
\begin{aligned}
\bar{W}_{k} & =\int_{0}^{t} \frac{s_{k}^{\phi}}{\cos ^{2}\left(\frac{\pi s_{k}^{\phi 2}}{2 k_{v}^{2}}\right)} \dot{s}_{k}^{\phi} d \tau \\
& =\int_{0}^{t} \frac{s_{k}^{\phi}}{\cos ^{2}\left(\frac{\pi s_{k}^{\phi 2}}{2 k_{v}^{2}}\right)}\left(\dot{s}_{k}-\operatorname{sgn}\left(s_{k}^{\phi}\right) \dot{\phi}\right) d \tau \\
& =\int_{0}^{t}\left(\alpha_{k} \dot{s}_{k}-\left|\alpha_{k}\right| \dot{\phi}\right) d \tau
\end{aligned}
$$

In terms of (8) and (27), one can obtain that

$$
\bar{W}_{k}=\int_{0}^{t}\left[\alpha_{k}\left(\bar{\lambda}^{T} \mathbf{e}_{k}+\dot{e}_{n, k}\right)-\left|\alpha_{k}\right| \dot{\phi}\right] d \tau
$$

Using the system model (1) and the desired state trajectory (3), (28) becomes

$$
\begin{aligned}
\bar{W}_{k} & =\int_{0}^{t}\left[\alpha_{k}\left(\bar{\lambda}^{T} \mathbf{e}_{k}+\dot{p}_{n, k}-\dot{x}_{n, k}\right)-\left|\alpha_{k}\right| \dot{\phi}\right] d \tau \\
& =\int_{0}^{t}\left[\alpha_{k}\left(\bar{\lambda}^{T} \mathbf{e}_{k}+h\left(\mathbf{p}_{k}\right)-\boldsymbol{\theta}^{T} \boldsymbol{\xi}\left(\mathbf{x}_{k}\right)-u_{k}-d_{k}\right)-\left|\alpha_{k}\right| \dot{\phi}\right] d \tau
\end{aligned}
$$


Substituting the control law (16) into (29) yields

$$
\begin{aligned}
\bar{W}_{k}= & \int_{0}^{t}\left[\alpha _ { k } \left(\bar{\lambda}^{T} \mathbf{e}_{k}-\tilde{\boldsymbol{\theta}}_{k}^{T} \boldsymbol{\xi}\left(\mathbf{x}_{k}\right)-\beta s_{k}-\left|\bar{\lambda}^{T} \mathbf{e}_{k}\right| \operatorname{sat}\left(\frac{s_{k}}{\phi}\right)\right.\right. \\
& \left.\left.+h\left(\mathbf{p}_{k}\right)-d_{k}-\hat{\rho}_{k} \operatorname{sat}\left(\frac{s_{k}}{\phi}\right)\right)-\left|\alpha_{k}\right| \dot{\phi}\right] d \tau
\end{aligned}
$$

From (14), one can derive that $\alpha_{k} \operatorname{sat}\left(\frac{s_{k}}{\phi}\right)=\left|\alpha_{k}\right|$. And with the definition of $\rho$ in (4), (30) can be rewritten as

$$
\begin{aligned}
\bar{W}_{k} \leq & \int_{0}^{t}\left[\alpha_{k} \bar{\lambda}^{T} \mathbf{e}_{k}-\alpha_{k} \tilde{\boldsymbol{\theta}}_{k}^{T} \boldsymbol{\xi}\left(\mathbf{x}_{k}\right)-\beta \alpha_{k} s_{k}\right. \\
& \left.-\left|\alpha_{k}\right|\left|\bar{\lambda}^{T} \mathbf{e}_{k}\right|+\left|\alpha_{k}\right|\left|h\left(\mathbf{p}_{k}\right)-d_{k}\right|-\left|\alpha_{k}\right| \hat{\rho}_{k}-\left|\alpha_{k}\right| \dot{\phi}\right] d \tau \\
\leq & \int_{0}^{t}\left[\alpha_{k} \bar{\lambda}^{T} \mathbf{e}_{k}-\alpha_{k} \tilde{\boldsymbol{\theta}}_{k}^{T} \boldsymbol{\xi}\left(\mathbf{x}_{k}\right)-\beta \alpha_{k} s_{k}\right. \\
& \left.-\left|\alpha_{k}\right|\left|\bar{\lambda}^{T} \mathbf{e}_{k}\right|+\left|\alpha_{k}\right| \tilde{\rho}_{k}-\left|\alpha_{k}\right| \dot{\phi}\right] d \tau
\end{aligned}
$$

In terms of (10) and the fact of $\alpha_{k} \bar{\lambda}^{T} \mathbf{e}_{k} \leq\left|\alpha_{k}\right|\left|\bar{\lambda}^{T} \mathbf{e}_{k}\right|$, one can obtain from (31) that

$$
\begin{aligned}
\bar{W}_{k} & \leq \int_{0}^{t}\left[-\alpha_{k} \tilde{\boldsymbol{\theta}}_{k}^{T} \boldsymbol{\xi}\left(\mathbf{x}_{k}\right)-\beta \alpha_{k}\left(s_{k}^{\phi}+\phi s a t\left(\frac{s_{k}}{\phi}\right)\right)+\left|\alpha_{k}\right| \tilde{\rho}_{k}-\left|\alpha_{k}\right| \dot{\phi}\right] d \tau \\
& =\int_{0}^{t}\left[-\alpha_{k} \tilde{\boldsymbol{\theta}}_{k}^{T} \boldsymbol{\xi}\left(\mathbf{x}_{k}\right)+\left|\alpha_{k}\right| \tilde{\rho}_{k}-\beta \alpha_{k} s_{k}^{\phi}-\left|\alpha_{k}\right| \beta \phi-\left|\alpha_{k}\right| \dot{\phi}\right] d \tau
\end{aligned}
$$

According to (12), (32) can be simplified as

$$
\bar{W}_{k} \leq \int_{0}^{t}\left[-\alpha_{k} \tilde{\boldsymbol{\theta}}_{k}^{T} \boldsymbol{\xi}\left(\mathbf{x}_{k}\right)+\left|\alpha_{k}\right| \tilde{\rho}_{k}-\beta \alpha_{k} s_{k}^{\phi}\right] d \tau
$$

Now let us examine the third and the fourth terms on the right side of equation (26). It is straightforward to derive that

$$
\begin{aligned}
\tilde{\bar{N}}_{k} \triangleq & \bar{N}_{k}-\bar{N}_{k-1} \\
= & \frac{1}{2} \int_{0}^{t}\left[\left(\boldsymbol{\theta}-\hat{\boldsymbol{\theta}}_{k}\right)^{T} \boldsymbol{\Lambda}^{-1}\left(\boldsymbol{\theta}-\hat{\boldsymbol{\theta}}_{k}\right)-\left(\boldsymbol{\theta}-\hat{\boldsymbol{\theta}}_{k-1}\right)^{T} \boldsymbol{\Lambda}^{-1}\left(\boldsymbol{\theta}-\hat{\boldsymbol{\theta}}_{k-1}\right)\right] d \tau \\
= & -\frac{1}{2} \int_{0}^{t}\left(2 \boldsymbol{\theta}-\hat{\boldsymbol{\theta}}_{k}-\hat{\boldsymbol{\theta}}_{k-1}\right)^{T} \boldsymbol{\Lambda}^{-1}\left(\hat{\boldsymbol{\theta}}_{k}-\hat{\boldsymbol{\theta}}_{k-1}\right) d \tau \\
= & -\int_{0}^{t}\left(\boldsymbol{\theta}-\hat{\boldsymbol{\theta}}_{k}\right)^{T} \boldsymbol{\Lambda}^{-1}\left(\hat{\boldsymbol{\theta}}_{k}-\hat{\boldsymbol{\theta}}_{k-1}\right) d \tau \\
& -\frac{1}{2} \int_{0}^{t}\left(\hat{\boldsymbol{\theta}}_{k}-\hat{\boldsymbol{\theta}}_{k-1}\right)^{T} \boldsymbol{\Lambda}^{-1}\left(\hat{\boldsymbol{\theta}}_{k}-\hat{\boldsymbol{\theta}}_{k-1}\right) d \tau
\end{aligned}
$$


According to the parameter learning laws (17) and (19), equation (34) leads to

$$
\begin{aligned}
\tilde{\bar{N}}_{k}= & -\int_{0}^{t}\left(\boldsymbol{\theta}-\operatorname{proj}_{\theta}\left(\hat{\boldsymbol{\theta}}_{k}^{*}\right)\right)^{T} \boldsymbol{\Lambda}^{-1}\left(\operatorname{proj}_{\theta}\left(\hat{\boldsymbol{\theta}}_{k}^{*}\right)-\hat{\boldsymbol{\theta}}_{k}^{*}-\boldsymbol{\Lambda} \boldsymbol{\xi}\left(\mathbf{x}_{k}\right) \alpha_{k}\right) d \tau \\
& -\frac{1}{2} \int_{0}^{t}\left(\hat{\boldsymbol{\theta}}_{k}-\hat{\boldsymbol{\theta}}_{k-1}\right)^{T} \boldsymbol{\Lambda}^{-1}\left(\hat{\boldsymbol{\theta}}_{k}-\hat{\boldsymbol{\theta}}_{k-1}\right) d \tau \\
= & \int_{0}^{t}\left(\boldsymbol{\theta}-\operatorname{proj}_{\theta}\left(\hat{\boldsymbol{\theta}}_{k}^{*}\right)\right)^{T} \boldsymbol{\Lambda}^{-1}\left(\hat{\boldsymbol{\theta}}_{k}^{*}-\operatorname{proj}_{\theta}\left(\hat{\boldsymbol{\theta}}_{k}^{*}\right)\right) d \tau+\int_{0}^{t}\left(\boldsymbol{\theta}-\hat{\boldsymbol{\theta}}_{k}\right)^{T} \boldsymbol{\xi}\left(\mathbf{x}_{k}\right) \alpha_{k} d \tau \\
& -\frac{1}{2} \int_{0}^{t}\left(\hat{\boldsymbol{\theta}}_{k}-\hat{\boldsymbol{\theta}}_{k-1}\right)^{T} \boldsymbol{\Lambda}^{-1}\left(\hat{\boldsymbol{\theta}}_{k}-\hat{\boldsymbol{\theta}}_{k-1}\right) d \tau
\end{aligned}
$$

By using (23) and (35), it is obvious that

$$
\tilde{\bar{N}}_{k} \leq \int_{0}^{t} \tilde{\boldsymbol{\theta}}_{k}^{T} \boldsymbol{\xi}\left(\mathbf{x}_{k}\right) \alpha_{k} d \tau-\frac{1}{2} \int_{0}^{t}\left(\hat{\boldsymbol{\theta}}_{k}-\hat{\boldsymbol{\theta}}_{k-1}\right)^{T} \boldsymbol{\Lambda}^{-1}\left(\hat{\boldsymbol{\theta}}_{k}-\hat{\boldsymbol{\theta}}_{k-1}\right) d \tau
$$

The latter two terms on the right side of equation (26) are further written as

$$
\begin{aligned}
\tilde{\bar{L}}_{k} & \triangleq \bar{L}_{k}-\bar{L}_{k-1} \\
& =\frac{1}{2 v} \int_{0}^{t}\left(\left(\rho-\hat{\rho}_{k}\right)^{2}-\left(\rho-\hat{\rho}_{k-1}\right)^{2}\right) d \tau \\
& =-\frac{1}{v} \int_{0}^{t}\left(\rho-\hat{\rho}_{k}\right)\left(\hat{\rho}_{k}-\hat{\rho}_{k-1}\right) d \tau-\frac{1}{2 v} \int_{0}^{t}\left(\hat{\rho}_{k}-\hat{\rho}_{k-1}\right)^{2} d \tau
\end{aligned}
$$

In terms of the parameter learning laws (18) and (20), (37) becomes

$$
\begin{aligned}
\tilde{\bar{L}}_{k}= & -\frac{1}{v} \int_{0}^{t}\left(\rho-\operatorname{proj}_{\rho}\left(\hat{\rho}_{k}^{*}\right)\right)\left(\operatorname{proj}_{\rho}\left(\hat{\rho}_{k}^{*}\right)-\hat{\rho}_{k}^{*}+v\left|\alpha_{k}\right|\right) d \tau \\
& -\frac{1}{2 v} \int_{0}^{t}\left(\hat{\rho}_{k}-\hat{\rho}_{k-1}\right)^{2} d \tau \\
= & \frac{1}{v} \int_{0}^{t}\left(\rho-\operatorname{proj}_{\rho}\left(\hat{\rho}_{k}^{*}\right)\right)\left(\hat{\rho}_{k}^{*}-\operatorname{proj}_{\rho}\left(\hat{\rho}_{k}^{*}\right)\right) d \tau \\
& -\int_{0}^{t} \tilde{\rho}_{k}\left|\alpha_{k}\right| d \tau-\frac{1}{2 v} \int_{0}^{t}\left(\hat{\rho}_{k}-\hat{\rho}_{k-1}\right)^{2} d \tau
\end{aligned}
$$

By using (24), (38) becomes

$$
\tilde{\bar{L}}_{k} \leq-\int_{0}^{t} \tilde{\rho}_{k}\left|\alpha_{k}\right| d \tau-\frac{1}{2 v} \int_{0}^{t}\left(\hat{\rho}_{k}-\hat{\rho}_{k-1}\right)^{2} d \tau
$$

Substituting (33), (36) and (39) into (26), and using the fact of $-\beta \alpha_{k} s_{k}^{\phi} \leq 0$, the difference $\Delta \bar{E}_{k}$ becomes negative definite as

$$
\begin{aligned}
\Delta \bar{E}_{k} \leq & -\int_{0}^{t} \beta \alpha_{k} s_{k}^{\phi} d \tau-\frac{1}{2 v} \int_{0}^{t}\left(\hat{\rho}_{k}-\hat{\rho}_{k-1}\right)^{2} d \tau-\bar{W}_{k-1} \\
& -\frac{1}{2} \int_{0}^{t}\left(\hat{\boldsymbol{\theta}}_{k}-\hat{\boldsymbol{\theta}}_{k-1}\right)^{T} \boldsymbol{\Lambda}^{-1}\left(\hat{\boldsymbol{\theta}}_{k}-\hat{\boldsymbol{\theta}}_{k-1}\right) d \tau \\
\leq & -\bar{W}_{k-1} \leq 0
\end{aligned}
$$


Part 2 of the proof of (T1). The boundedness of $B C E F \bar{E}_{k}(t)$ and the convergence of $s_{k}^{\phi}(t)$

According to (40), $\bar{E}_{k}$ is a non-increasing sequence. Thus if $\bar{E}_{0}$ is bounded, one can conclude that $\bar{E}_{k}$ is bounded. Next, we will show the boundedness of $\bar{E}_{0}$. From (25) with $k=0$, one can derive that

$$
\bar{E}_{0}=\frac{k_{v}^{2}}{\pi} \tan \left(\frac{\pi s_{0}^{\phi 2}}{2 k_{v}^{2}}\right)+\frac{1}{2} \int_{0}^{t} \tilde{\boldsymbol{\theta}}_{0}^{T}(\tau) \Lambda^{-1} \tilde{\boldsymbol{\theta}}_{0}(\tau) d \tau+\frac{1}{2 v} \int_{0}^{t} \tilde{\rho}_{0}^{2}(\tau) d \tau
$$

According to (27) and (33), and using the fact of $-\beta \alpha_{0} s_{0}^{\phi} \leq 0$, the derivative of $\bar{E}_{0}$ is

$$
\begin{aligned}
\dot{\bar{E}}_{0} & =\frac{s_{0}^{\phi}}{\cos ^{2}\left(\frac{\pi s_{0}^{\phi 2}}{2 k_{v}^{2}}\right)} \dot{s}_{0}^{\phi}+\frac{1}{2} \tilde{\boldsymbol{\theta}}_{0}^{T} \boldsymbol{\Lambda}^{-1} \tilde{\boldsymbol{\theta}}_{0}+\frac{1}{2 v} \tilde{\rho}_{0}^{2} \\
& \leq-\alpha_{0} \xi^{T}\left(\mathbf{x}_{0}\right) \tilde{\boldsymbol{\theta}}_{0}+\left|\alpha_{0}\right| \tilde{\rho}_{0}+\frac{1}{2} \tilde{\boldsymbol{\theta}}_{0}^{T} \boldsymbol{\Lambda}^{-1} \tilde{\boldsymbol{\theta}}_{0}+\frac{1}{2 v} \tilde{\rho}_{0}^{2}
\end{aligned}
$$

In terms of the parameter learning laws (17) and (18), one can obtain from (42) that

$$
\dot{\bar{E}}_{0} \leq\left(\hat{\boldsymbol{\theta}}_{0}^{*}-\hat{\boldsymbol{\theta}}_{-1}\right)^{T} \boldsymbol{\Lambda}^{-1} \tilde{\boldsymbol{\theta}}_{0}+\frac{1}{2} \tilde{\boldsymbol{\theta}}_{0}^{T} \boldsymbol{\Lambda}^{-1} \tilde{\boldsymbol{\theta}}_{0}+\frac{1}{v}\left(\hat{\rho}_{0}^{*}-\hat{\rho}_{-1}\right) \tilde{\rho}_{0}+\frac{1}{2 v} \tilde{\rho}_{0}^{2}
$$

According to (19), (20), and (43), one can get that

$$
\begin{aligned}
\dot{\bar{E}}_{0} & \leq \hat{\boldsymbol{\theta}}_{0}^{* T} \boldsymbol{\Lambda}^{-1} \tilde{\boldsymbol{\theta}}_{0}+\tilde{\boldsymbol{\theta}}_{0}^{T} \boldsymbol{\Lambda}^{-1} \tilde{\boldsymbol{\theta}}_{0}+\frac{1}{v} \hat{\rho}_{0}^{*} \tilde{\rho}_{0}+\frac{1}{v} \tilde{\rho}_{0}^{2} \\
& =\hat{\boldsymbol{\theta}}_{0}^{* T} \boldsymbol{\Lambda}^{-1} \tilde{\boldsymbol{\theta}}_{0}+\left(\boldsymbol{\theta}-\hat{\boldsymbol{\theta}}_{0}\right)^{T} \boldsymbol{\Lambda}^{-1} \tilde{\boldsymbol{\theta}}_{0}+\frac{1}{v} \hat{\rho}_{0}^{*} \tilde{\rho}_{0}+\frac{1}{v}\left(\rho-\hat{\rho}_{0}\right) \tilde{\rho}_{0}
\end{aligned}
$$

By using (23) and (24) of Lemma 1, (44) can be simplified as follows

$$
\begin{aligned}
\dot{\bar{E}}_{0} \leq & \left(\hat{\boldsymbol{\theta}}_{0}^{*}-\operatorname{proj}_{\theta}\left(\hat{\boldsymbol{\theta}}_{0}^{*}\right)\right)^{T} \boldsymbol{\Lambda}^{-1}\left(\boldsymbol{\theta}-\operatorname{proj}_{\theta}\left(\hat{\boldsymbol{\theta}}_{0}^{*}\right)\right)+\boldsymbol{\theta}^{T} \boldsymbol{\Lambda}^{-1} \tilde{\boldsymbol{\theta}}_{0} \\
& +\frac{1}{v}\left(\hat{\rho}_{0}^{*}-\operatorname{proj}_{\rho}\left(\hat{\rho}_{0}^{*}\right)\right)\left(\rho-\operatorname{proj}_{\rho}\left(\hat{\rho}_{0}^{*}\right)\right)+\frac{1}{v} \rho \tilde{\rho}_{0} \\
\leq & \boldsymbol{\theta}^{T} \boldsymbol{\Lambda}^{-1} \tilde{\boldsymbol{\theta}}_{0}+\frac{1}{v} \rho \tilde{\rho}_{0}
\end{aligned}
$$

By Young's inequality [37], it is derived from (45) that

$$
\dot{\bar{E}}_{0} \leq c_{1} \lambda_{\max }\left(\boldsymbol{\Lambda}^{-1}\right)\left\|\tilde{\boldsymbol{\theta}}_{0}\right\|^{2}+\frac{1}{4 c_{1}}\|\boldsymbol{\theta}\|^{2}+\frac{1}{v} c_{2}\left|\tilde{\rho}_{0}\right|^{2}+\frac{1}{4 c_{2}} \rho^{2}
$$

where $c_{1}, c_{2}$ are positive constants. 
According to Lemma $1, \boldsymbol{\theta}$ and $\rho$ are bounded for all $t \in[0, T] . \tilde{\boldsymbol{\theta}}_{0}$ and $\tilde{\rho}_{0}$ are also bounded by virtue of the projection mechanism. Thus, $\dot{\bar{E}}_{0}$ is bounded over $[0, T]$ which implies the continuity of $\bar{E}_{0}$. Then, it is straightforward to derive that $\bar{E}_{0}$ is bounded for $\forall t \in[0, T]$ because it is continuous [37]. As a result, the boundedness of $\bar{E}_{k}$ is guaranteed accordingly.

Next, the convergence of $s_{k}^{\phi}(t)$ will be proved.

Summing (40) from 1 to $k$ yields

$$
\begin{gathered}
\bar{E}_{k} \leq \bar{E}_{0}-\sum_{j=1}^{k} \bar{W}_{j-1}=\bar{E}_{0}-\sum_{j=1}^{k} \frac{k_{v}^{2}}{\pi} \tan \left(\frac{\pi s_{j-1}^{\phi 2}}{2 k_{v}^{2}}\right) \\
\lim _{k \rightarrow \infty} \bar{E}_{k} \leq \bar{E}_{0}-\lim _{k \rightarrow \infty} \sum_{j=1}^{k} \frac{k_{v}^{2}}{\pi} \tan \left(\frac{\pi s_{j-1}^{\phi 2}}{2 k_{v}^{2}}\right)
\end{gathered}
$$

Based on inequality (48), since $\bar{E}_{0}$ and $\bar{E}_{k}$ are bounded, one can derive that the infinite series $\sum_{j=1}^{\infty} \frac{k_{v}^{2}}{\pi} \tan \left(\frac{\pi s_{j-1}^{\phi 2}}{2 k_{v}^{2}}\right)$ converges. Hence, $s_{k}^{\phi}(t)$ converges to zero asymptotically along the iteration axis for all $t \in[0, T]$, namely $\lim _{k \rightarrow \infty} s_{k}^{\phi}(t)=0$.

(T2) It is derived from (12) that $0<\delta \exp (-\beta T) \leq \phi(t) \leq \delta$. Then, in terms of equation (10) and the conclusion of (T1), one can obtain that for $\forall t \in[0, T]$

$$
\begin{aligned}
\lim _{k \rightarrow \infty}\left|s_{k}(t)\right| & =\lim _{k \rightarrow \infty}\left|s_{k}^{\phi}(t)+\phi(t) \operatorname{sat}\left(\frac{s_{k}(t)}{\phi(t)}\right)\right| \\
& \leq \lim _{k \rightarrow \infty}\left|s_{k}^{\phi}(t)\right|+\phi(t)=\phi(t) \leq \delta
\end{aligned}
$$

(T3) The proof consists of two parts. Part 1 derives the convergence property of the tracking error $e_{1, k}(t)$. Part 2 proves the convergence of the tracking errors $e_{j, k}(t), j=2, \ldots, n$.

Part 1 of the proof of (T3). The convergence of the tracking error $e_{1, k}$

Define

$$
s_{k}=\left(\frac{d}{d t}+c\right)^{n-1} e_{1, k}
$$


where $c$ is a strictly positive constant. Taking $n=3$, for example,

$$
\begin{aligned}
s_{k} & =\left(\frac{d}{d t}+c\right)^{2} e_{1, k}=\left[\frac{d^{2}}{d t^{2}}+2 c \frac{d}{d t}+c^{2}\right] e_{1, k} \\
& =\ddot{e}_{1, k}+2 c \dot{e}_{1, k}+c^{2} e_{1, k}=e_{1, k}^{(2)}+2 c e_{1, k}^{(1)}+c^{2} e_{1, k}=e_{3, k}+2 c e_{2, k}+c^{2} e_{1, k}
\end{aligned}
$$

According to equation (8), for $n=3$,

$$
s_{k}=e_{3, k}+\lambda_{2} e_{2, k}+\lambda_{1} e_{1, k}
$$

Hence, the tunable controller parameters $\lambda_{1}, \lambda_{2}$ are related to $c$ such that $\lambda_{1}=c^{2}$ and $\lambda_{2}=2 c$.

According to this definition, the tracking error $e_{1, k}$ is obtained from $s_{k}$ through a sequence of first-order lowpass filters as shown in Fig.1 [43].

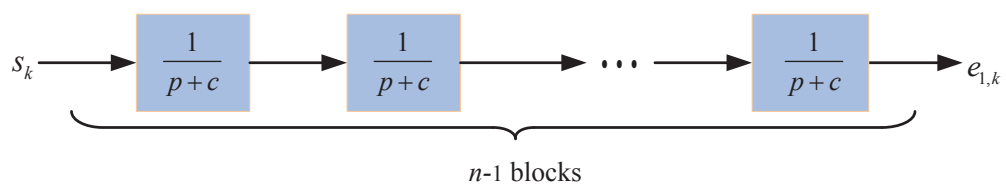

Figure 1: Computing bound on $e_{1, k}(p=d / d t)$.

By solving a series of first-order differential equations based on Fig.1, and according to (T2) that $\lim _{k \rightarrow \infty}\left|s_{k}(t)\right| \leq \phi(t) \leq \delta, \forall t \in[0, T]$, one can derive that

$$
\lim _{k \rightarrow \infty}\left|e_{1, k}\right| \leq\left[\frac{\delta}{c^{n-1}}+\lim _{k \rightarrow \infty} \sum_{z=1}^{n-1} \frac{\left.\left[(p+c)^{n-z-1}\left|e_{1, k}(t)\right|\right]\right|_{t=0}}{c^{n-z-1}}\right]
$$

where $p=d / d t$. For $z=n-1$,

$$
\left.\left[(p+c)^{n-z-1}\left|e_{1, k}(t)\right|\right]\right|_{t=0}=\left|e_{1, k}(0)\right| \leq \delta_{1}
$$

and for $z=n-2$,

$$
\left.\left[(p+c)^{n-z-1}\left|e_{1, k}(t)\right|\right]\right|_{t=0}=\left|e_{2, k}(0)\right|+c\left|e_{1, k}(0)\right| \leq \delta_{2}+c \delta_{1}
$$

The same reasoning can be applied for $z=1,2, \ldots, n-3$.

In the subsequent Remark 4, an example will be given to explain how inequality (51) is derived in detail.

Part 2 of the proof of (T3). The convergence of the tracking errors $e_{j, k}, j=2, \ldots, n$

Similarly, the tracking error $e_{1, k}^{(i)}, i=0,1, \ldots, n-1$, can be obtained through the sequence of Fig.2 [43]. Note that $\frac{p}{p+c}=1-\frac{c}{p+c}$. Hence, in terms of (51), Fig.2 implies 


$$
\lim _{k \rightarrow \infty}\left|e_{1, k}^{(i)}\right| \leq\left[\frac{\delta}{c^{n-1-i}}+\sigma_{i}\right]\left(1+\frac{c}{c}\right)^{i}, i=0,1, \ldots, n-1
$$

where $\sigma_{i} \triangleq \lim _{k \rightarrow \infty} \sum_{z=1}^{n-1-i} \frac{\left.\left[\left(\frac{d}{d t}+c\right)^{n-z-1}\left|e_{1, k}(t)\right|\right]\right|_{t=0}}{c^{n-z-i-1}}$. Denote $\sum_{z=l}^{q}(\cdot)_{z} \triangleq 0$ for $l>q$.

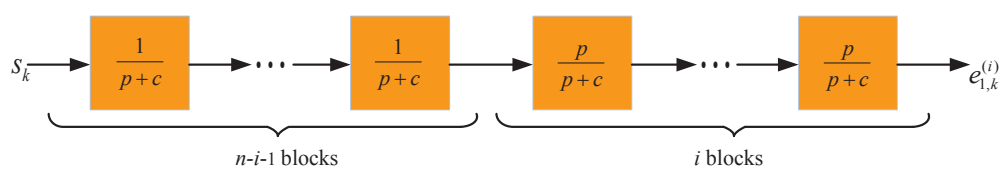

Figure 2: Computing bounds on $e_{1, k}^{(i)}(i=0,1, \ldots, n-1 \cdot p=d / d t)$.

(T4) This proof will directly derive the bounds of the state $\mathbf{x}_{k}(t)$ and input $u_{k}(t)$ at first, and then guarantee both state and input constraints for any time in all iterations. According to (T1), $\bar{E}_{k}$ is bounded for any iteration such that the term $\bar{W}_{k} \triangleq \frac{k_{v}^{2}}{\pi} \tan$ $\left(\frac{\pi s_{k}^{\phi 2}}{2 k_{v}^{2}}\right)$ in $\bar{E}_{k}$ is also bounded for any iteration. Therefore, with the facts that $\bar{W}_{k}$ is bounded and $s_{k}^{\phi}(0)=0$, it implies $\left|s_{k}^{\phi}\right|<k_{v}$ for all time and iterations. According to (10), one can easily get that $\left|s_{k}\right|<k_{v}+\delta$.

Analogous to the analysis from (51) to (52) in (T3), the bounds of state errors can be derived as

$$
\left|e_{1, k}^{(i)}\right|<\left[\frac{k_{v}+\delta}{c^{n-1-i}}+\sigma_{i}\right] 2^{i}, i=0,1, \ldots, n-1
$$

In terms of (2) and the definition of the state error $e_{j, k}=p_{j, k}-x_{j, k}, j=1,2, \ldots, n$, the states of the system are bounded by

$$
\left|x_{i+1, k}\right|<\left[\frac{k_{v}+\delta}{c^{n-1-i}}+\sigma_{i}\right] 2^{i}+\eta_{i+1}, i=0,1, \ldots, n-1
$$

Using the triangular inequality, the control input based on (16) is bounded by

$$
\begin{aligned}
\left|u_{k}(t)\right| \leq & \beta\left|s_{k}(t)\right|+\sum_{i=1}^{n-1} \lambda_{i}\left|e_{1, k}^{(i)}\right|+\sum_{z=1}^{m} \max \left\{\left|\theta_{z, \min }\right|,\left|\theta_{z, \max }\right|\right\} \cdot\left|\xi_{z}\right|+\rho_{\max } \\
< & \beta\left(k_{v}+\delta\right)+\sum_{i=1}^{n-1} \lambda_{i}\left[\frac{k_{v}+\delta}{c^{n-1-i}}+\sigma_{i}\right] 2^{i} \\
& +\sum_{z=1}^{m} \max \left\{\left|\theta_{z, \min }\right|,\left|\theta_{z, \max }\right|\right\} \cdot\left|\xi_{z}\right|+\rho_{\max }
\end{aligned}
$$

If we want to retain the input $u_{k}$ and the states $x_{j, k}, j=1,2, \ldots, n$ within the input constraint $u_{\text {max }}$ and state constraints $x_{j, \max }, j=1,2, \ldots, n$, we can select $k_{v}, c, \beta$ 
such that the following two inequalities are satisfied:

$$
\left[\frac{k_{v}+\delta}{c^{n-1-i}}+\sigma_{i}\right] 2^{i}+\eta_{i+1} \leq x_{i+1, \max }, i=0,1, \ldots, n-1
$$

and

$$
\begin{aligned}
& \beta\left(k_{v}+\delta\right)+\sum_{i=1}^{n-1} \lambda_{i}\left[\frac{k_{v}+\delta}{c^{n-1-i}}+\sigma_{i}\right] 2^{i} \\
& +\sum_{z=1}^{m} \max \left\{\left|\theta_{z, \min }\right|,\left|\theta_{z, \max }\right|\right\} \cdot\left|\xi_{z}\right|+\rho_{\max } \leq u_{\max }
\end{aligned}
$$

Then, the input constraint (5) and state constraints (6) can always be satisfied.

This completes the proof.

Remark 3. It is worth pointing out that $\bar{W}_{k} \triangleq \frac{k_{v}^{2}}{\pi} \tan \left(\frac{\pi s_{k}^{\phi 2}}{2 k_{v}^{2}}\right)$ in the defined BCEF (25) is a Barrier Lyapunov Function (BLF) [44-46]. It will go to infinity as the argument $\left|s_{k}^{\phi}\right|$ approaches the limit $k_{v}$. However, in Part 2 of the proof of (T1), the boundedness of the $\mathrm{BCEF} \bar{E}_{k}$ will guarantee the boundedness of the BLF $\bar{W}_{k}$ for any iteration. Therefore, the constraint $\left|s_{k}^{\phi}\right|<k_{v}$ will be ensured for any time in all iterations. Based on this fact, (T4) proved in detail that how the proposed AILC guarantees both the state and input signals within their given constrained ranges.

Remark 4. Let us take $n=3$ as an example to see how inequality (51) is derived. According to (50) with $n=3$, the tracking error $e_{1, k}$ will be obtained from $s_{k}$ through two first-order lowpass filters as shown in Fig.3, i.e., $s_{k}\left(\frac{1}{p+c}\right)^{2}=e_{1, k}$.

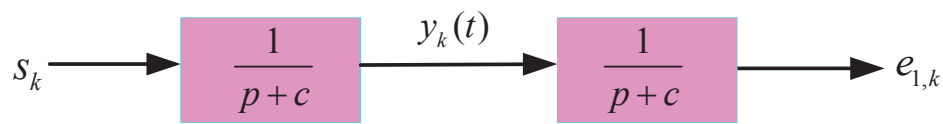

Figure 3: Computing bound on $e_{1, k}$ with $n=3(p=d / d t)$.

Let $y_{k}(t)$ be the output of the first filter, then one can obtain that $(p+c) y_{k}=s_{k}$, i.e.,

$$
\dot{y}_{k}+c y_{k}=s_{k}
$$

Solving the above first-order differential equation (58), one has

$$
y_{k}(t)=\int_{0}^{t} s_{k}(\tau) \mathrm{e}^{-c(t-\tau)} d \tau+y_{k}(0) \mathrm{e}^{-c t}
$$

Since $\lim _{k \rightarrow \infty}\left|s_{k}(t)\right| \leq \delta$ according to (T2), one can get 


$$
\begin{aligned}
\lim _{k \rightarrow \infty}\left|y_{k}(t)\right| & \leq \delta \int_{0}^{t} \mathrm{e}^{-c(t-\tau)} d \tau+\lim _{k \rightarrow \infty}\left|y_{k}(0)\right| \mathrm{e}^{-c t} \\
& =\frac{\delta}{c}\left(1-\mathrm{e}^{-c t}\right)+\lim _{k \rightarrow \infty}\left|y_{k}(0)\right| \mathrm{e}^{-c t} \leq \frac{\delta}{c}+\lim _{k \rightarrow \infty}\left|y_{k}(0)\right|
\end{aligned}
$$

Based on the second filter, it is derived that

$$
y_{k}=(p+c) e_{1, k}=\dot{e}_{1, k}+c e_{1, k}=e_{2, k}+c e_{1, k}
$$

By virtue of (61), one has

$$
y_{k}(0)=\left.(p+c) e_{1, k}(0) \triangleq\left[(p+c) e_{1, k}(t)\right]\right|_{t=0}=e_{2, k}(0)+c e_{1, k}(0)
$$

and

$$
\left|y_{k}(0)\right| \leq\left|e_{2, k}(0)\right|+\left.c\left|e_{1, k}(0)\right| \triangleq\left[(p+c)\left|e_{1, k}(t)\right|\right]\right|_{t=0}
$$

According to (63), and based on (7) in Assumption A1 that the initial state errors are bounded, (60) leads to

$$
\lim _{k \rightarrow \infty}\left|y_{k}(t)\right| \leq \frac{\delta}{c}+\left.\lim _{k \rightarrow \infty}\left[(p+c)\left|e_{1, k}(t)\right|\right]\right|_{t=0} \leq \frac{\delta}{c}+\delta_{2}+c \delta_{1}
$$

Then, by solving the differential equation (61), one can derive that

$$
e_{1, k}(t)=\int_{0}^{t} y_{k}(\tau) \mathrm{e}^{-c(t-\tau)} d \tau+e_{1, k}(0) \mathrm{e}^{-c t}
$$

According to (64), (65) can be rewritten as

$$
\begin{aligned}
\lim _{k \rightarrow \infty}\left|e_{1, k}\right| \leq & \left\{\frac{\delta}{c}+\left.\lim _{k \rightarrow \infty}\left[(p+c)\left|e_{1, k}(t)\right|\right]\right|_{t=0}\right\} \int_{0}^{t} \mathrm{e}^{-c(t-\tau)} d \tau \\
& +\lim _{k \rightarrow \infty}\left|e_{1, k}(0)\right| \mathrm{e}^{-c t}
\end{aligned}
$$

Analogous to the analysis of (60), (66) can be simplified as

$$
\begin{aligned}
\lim _{k \rightarrow \infty}\left|e_{1, k}\right| & \leq \frac{\delta}{c^{2}}+\frac{\left.\lim _{k \rightarrow \infty}\left[(p+c)\left|e_{1, k}(t)\right|\right]\right|_{t=0}}{c}+\lim _{k \rightarrow \infty}\left|e_{1, k}(0)\right| \\
& =\frac{\delta}{c^{2}}+\lim _{k \rightarrow \infty} \sum_{z=1}^{2} \frac{\left.\left[(p+c)^{2-z}\left|e_{1, k}(t)\right|\right]\right|_{t=0}}{c^{2-z}}
\end{aligned}
$$

Consequently, inequality (51) is derived. 


\section{Simulation Results}

A train operation system similar to CRH2-A is simulated to demonstrate the validity and applicability of the proposed AILC.

According to Newton's law, the most widely used dynamic model of a train $[47,48]$ can be shown as follows:

$$
\begin{aligned}
& \dot{x}_{1, k}(t)=x_{2, k}(t) \\
& \dot{x}_{2, k}(t)=u_{k}(t)-f_{a}(t)-f_{b}(t)+d_{k}(t)
\end{aligned}
$$

where $x_{1, k}(t)(\mathrm{m})$ and $x_{2, k}(t)(\mathrm{m} / \mathrm{s})$ denote the real-time position and speed of the train; the control input $u_{k}(t)(\mathrm{N} / \mathrm{kg})$ is the traction force (or braking force when it becomes negative) on unit mass. $d_{k}(t)(\mathrm{N} / \mathrm{kg})$ denotes the unknown load disturbance on unit mass which is caused by different numbers of passengers/loads in each operation. $f_{a}(t)$ $(\mathrm{N} / \mathrm{kg})$ represents the additional resistance on unit mass. It has an influence on certain segments like curves, ramps, and tunnels. And, it is an unknown and time-varying function. $f_{b}(t)(\mathrm{N} / \mathrm{kg})$ denotes the basic resistance on unit mass which consists of mechanical resistance $a(t)+b(t) x_{2, k}(t)$ and air resistance $c(t) x_{2, k}{ }^{2}(t)$, and can be represented as $f_{b}(t)=a(t)+b(t) x_{2, k}(t)+c(t) x_{2, k}{ }^{2}(t)$, where $a(t), b(t)$, and $c(t)$ are unknown and time-varying resistance coefficients.

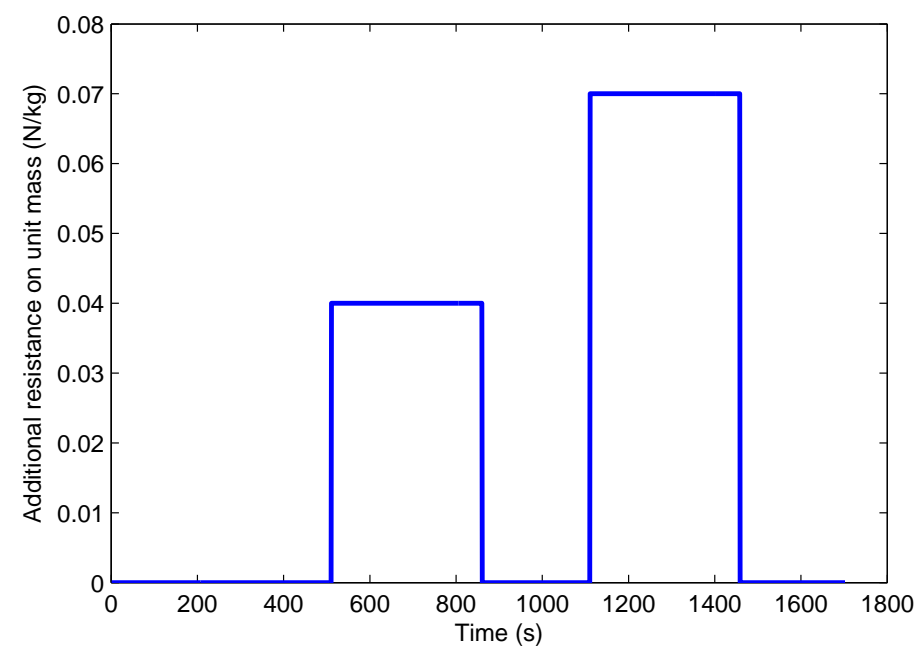

Figure 4: The additional resistance on unit mass along the track.

Consequently, the unknown parameter vector in (68) is $\boldsymbol{\theta}(t)=[a(t), b(t), c(t)$, $\left.f_{a}(t)\right]^{T}$, and the corresponding known state-dependent vector function $\boldsymbol{\xi}\left(x_{2, k}(t)\right)=$ $\left[-1,-x_{2, k}(t),-x_{2, k}^{2}(t),-1\right]^{T}$.

The railway track is $71.62 \mathrm{~km}$ between departure station and terminal station, with one ramp and one curve. The time interval of the simulation is 1700 seconds. The sampling time is 1 second. The total nominal mass of the train is $M=345000(\mathrm{~kg})$ 
at each iteration. The load disturbance on unit mass $d_{k}(t)$ is randomly and iteratively varying within $[-0.01,0.01](\mathrm{N} / \mathrm{kg})$, due to the change of the passengers/loads in each operation.

The additional resistance $f_{a}(t)$ undergone by the train is shown in Fig. 4. The actual resistance coefficients $a(t), b(t)$ and $c(t)$ are satisfying:

$$
\begin{aligned}
& a(t)=[2977+275 \sin (0.0039 t)] / M \\
& b(t)=[25.17+2.4 \sin (0.0039 t)] / M \\
& c(t)=\frac{273.15}{273.15+25}(0.3864+0.03 \sin (0.0039 t)) / M
\end{aligned}
$$

The desired position and speed trajectories $p_{1}(t)$ and $p_{2}(t)$ are depicted in Fig. 5, and the change in the desired speed trajectory is caused by the influence of the additional resistance $f_{a}(t)$. The initial position error $e_{1, k}(0)=p_{1}(0)-x_{1, k}(0)$ is randomly and iteratively varying within the interval $[0,0.05](\mathrm{m})$, and the initial velocity error $e_{2, k}(0)=p_{2}(0)-x_{2, k}(0)=0(\mathrm{~m} / \mathrm{s})$.
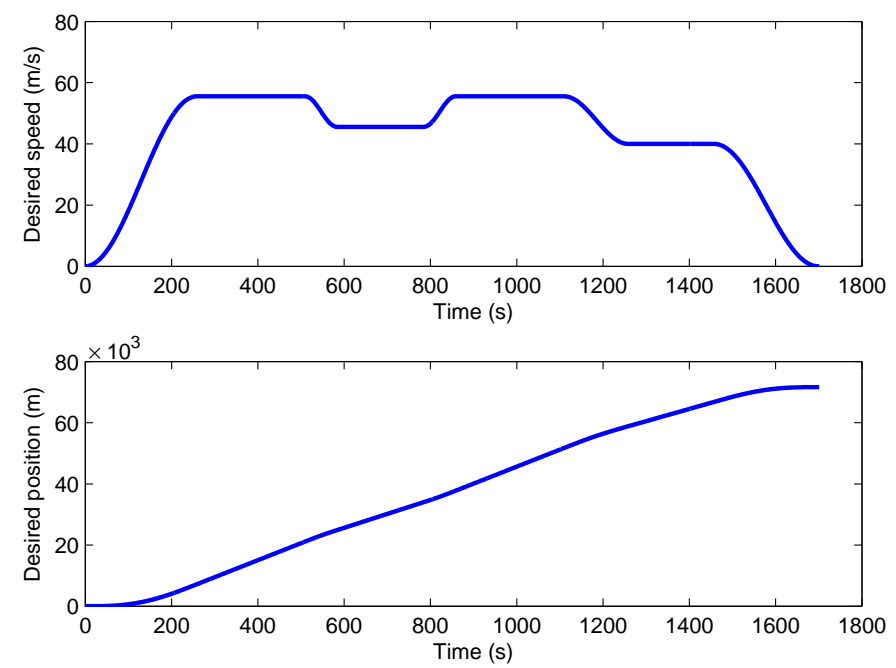

Figure 5: Desired speed and position trajectories.

The speed constraint of CRH2-A is set to be $x_{2, \max }=210 / 3.6(\mathrm{~m} / \mathrm{s})$. The train is operating with desired speed under 200/3.6 (m/s) for the whole track. According to the traction characteristic curve of CRH2-A [49], the maximum traction force under 200/3.6 (m/s) approximately satisfies

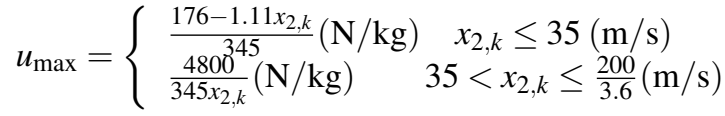

and the maximum braking force on unit mass is set to be $u_{\min }=-0.45(\mathrm{~N} / \mathrm{kg})$.

The objective of this train operation task is to make the actual speed and position track their desired ones, while the speed and input force signals are always within their maximum limits. 


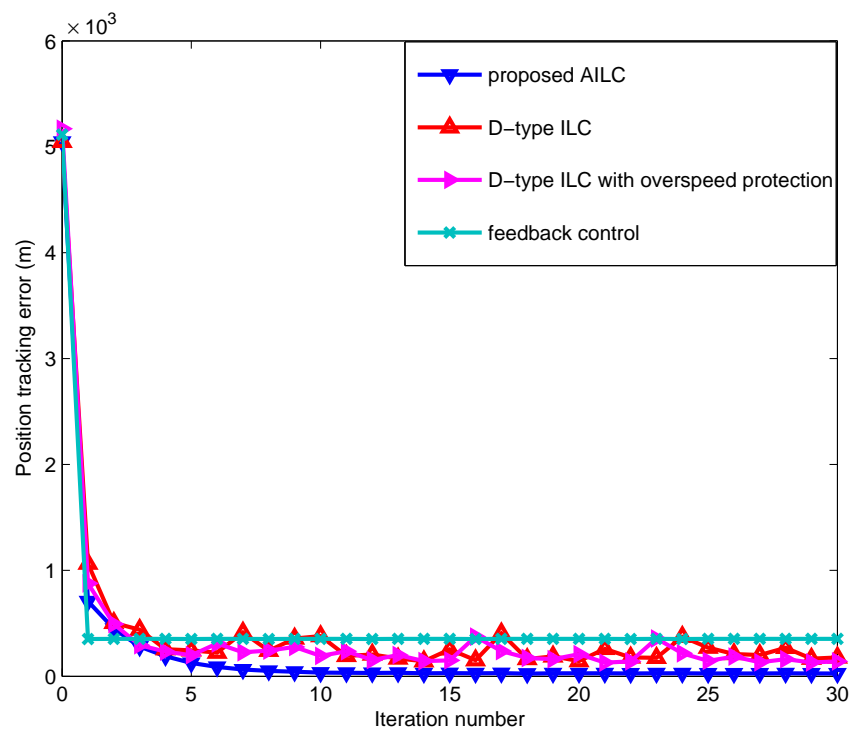

Figure 6: Position tracking errors.

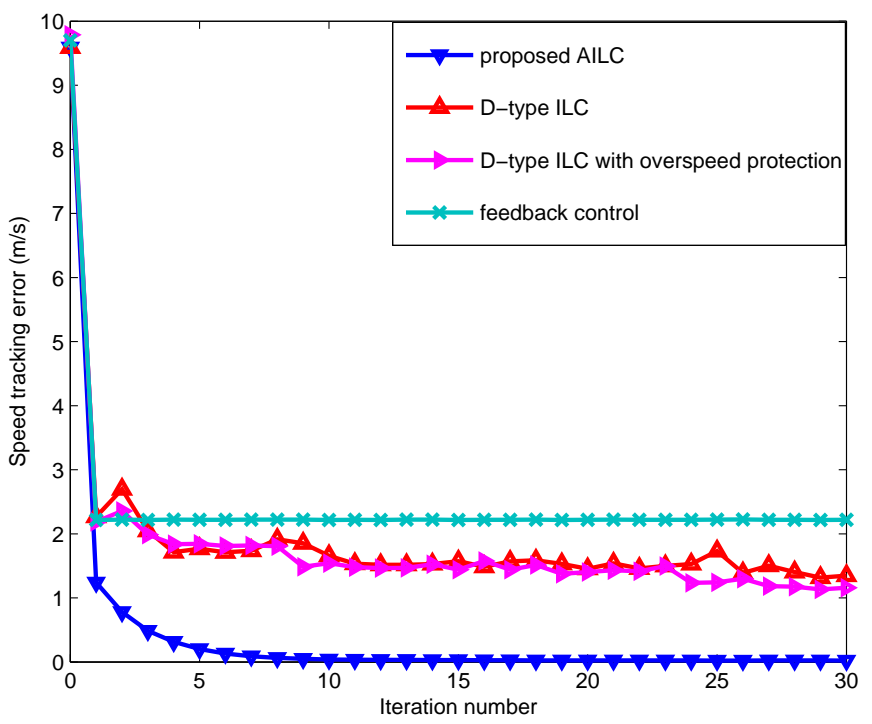

Figure 7: Speed tracking errors.

For comparison purposes, a typical pure D-type ILC scheme [50], a D-type ILC scheme with overspeed protection [6], a pure feedback control scheme and the pro- 


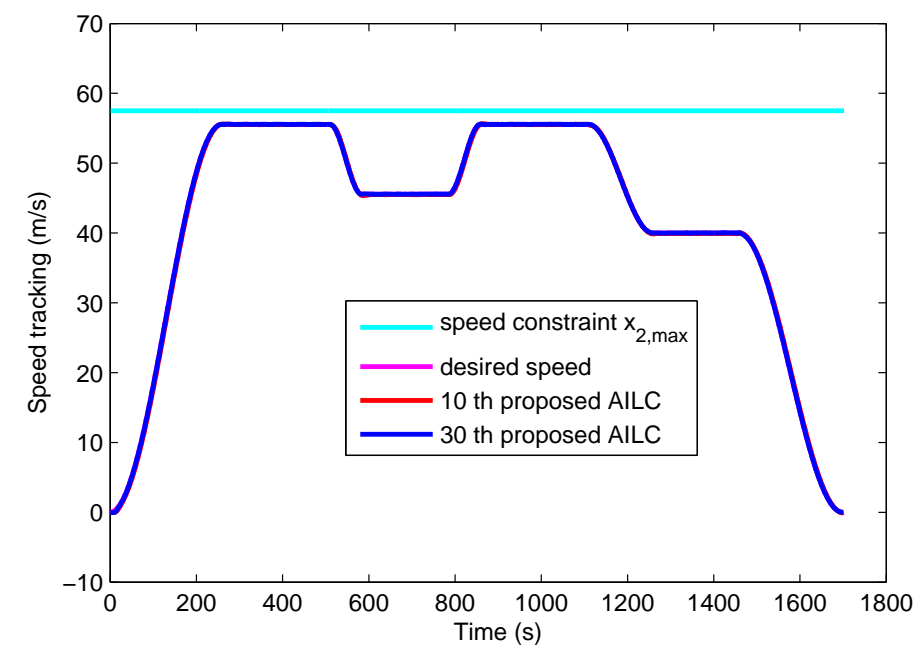

Figure 8: Speed tracking profile of the proposed AILC at the 10th and 30th iterations.

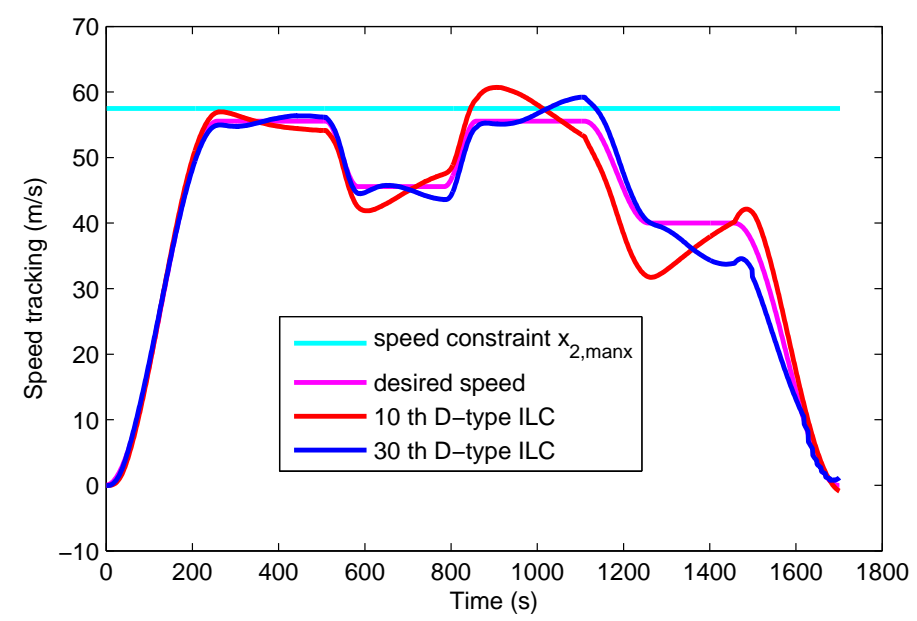

Figure 9: Speed tracking profile of the D-type ILC at the 10th and 30th iterations.

posed AILC scheme are all applied to the train operation system.

(1) The proposed AILC: The control parameters of the proposed AILC are selected as: $n=2, \beta=0.06, \lambda_{1}=0.005, \delta=0.5 . \Lambda=\operatorname{diag}\{0.02,0.02,0.02,0.02\}$ and $v=$ $6 \times 10^{-4} . \quad k_{v}=0.05 . \quad \hat{\boldsymbol{\theta}}_{k}(0)=[0,0,0,0]^{T}$. The lower and upper bounds for $\hat{\boldsymbol{\theta}}_{k}(t)$ and $\hat{\rho}_{k}$ are $\boldsymbol{\theta}_{\min }=\left[0,1 \times 10^{-5}, 1 \times 10^{-7}, 0\right]^{T}, \boldsymbol{\theta}_{\max }=\left[0.01,1 \times 10^{-4}, 4 \times 10^{-6}, 0.1\right]^{T}$, $\rho_{\text {min }}=0.01$, and $\rho_{\max }=0.05$.

(2) D-type ILC: A typical pure D-type ILC scheme in [50] is applied, which has 


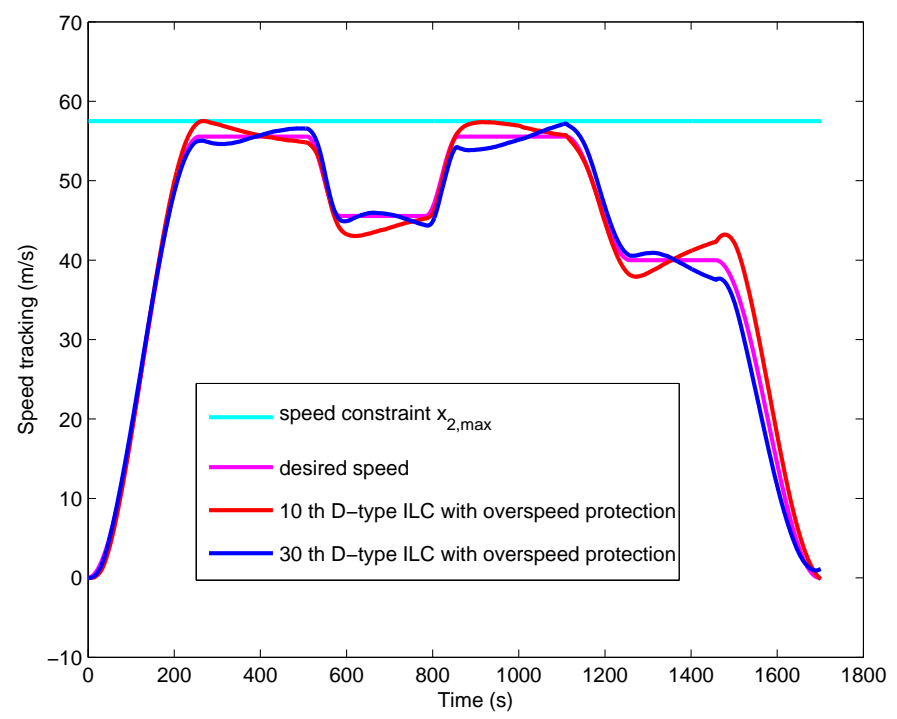

Figure 10: Speed tracking profile of the D-type ILC with overspeed protection at the 10th and 30th iterations.

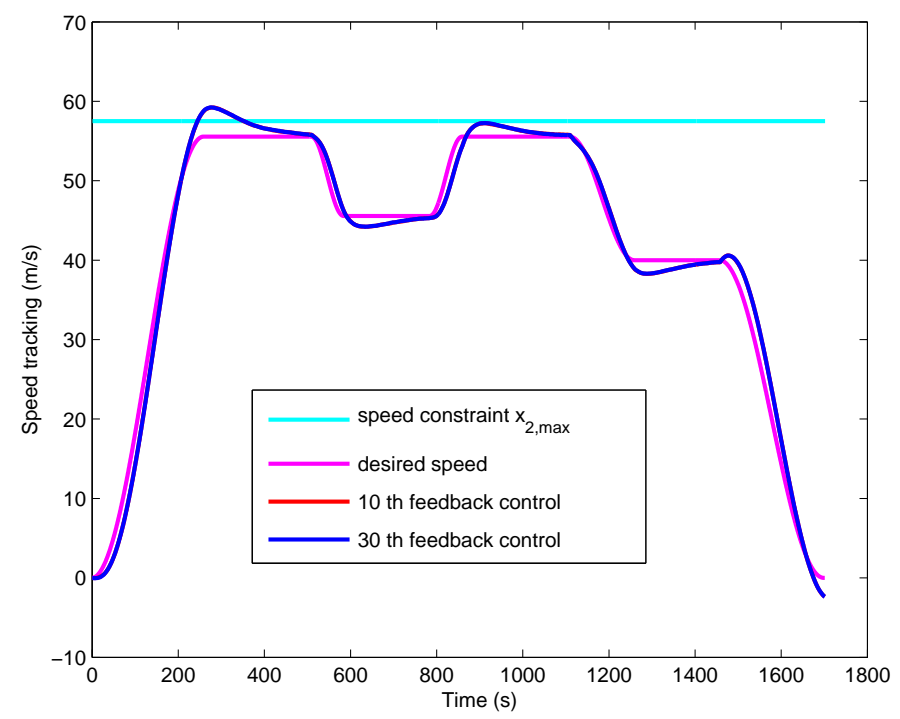

Figure 11: Speed tracking profile of the feedback control at the 10th and 30th iterations.

been shown to be robust to unknown disturbances and initial state errors:

$$
u_{k+1}(t)=u_{k}(t)+\mathbf{L}\left[\begin{array}{c}
\dot{e}_{1, k}(t) \\
\dot{e}_{2, k}(t)
\end{array}\right]
$$


together with the initial state learning laws given by

$$
x_{k+1}(0)=x_{k}(0)+\mathbf{B L}\left[\begin{array}{c}
\dot{e}_{1, k}(0) \\
\dot{e}_{2, k}(0)
\end{array}\right]
$$

where $\mathbf{B}=\left[\begin{array}{ll}0 & 1\end{array}\right]^{T}$ is the input distribution matrix. $\mathbf{L}=\left[L_{1}, L_{2}\right]$ is a constant learning gain vector. Based on intensive tuning, best control performance is obtained with $L_{1}=$ 0.002 , and $L_{2}=0.01$.

(3) D-type ILC with overspeed protection: According to [6], an overspeed penalty term is added to the pure D-type ILC scheme (71) to cope with state constraint:

$$
u_{k+1}(t)=u_{k}(t)+\mathbf{L}\left[\begin{array}{c}
\dot{e}_{1, k}(t) \\
\dot{e}_{2, k}(t)
\end{array}\right]-\ell \cdot \max \left[x_{2, k}(t)-x_{2, \max }, 0\right]
$$

where $\ell$ is a penalty gain of overspeed and $x_{2, \max }$ is the speed constraint. The added overspeed penalty term will play an important role when the actual operation speed $x_{2, k}(t)$ exceeds the speed constraint $x_{2, \max }$, and $\ell$ should be carefully selected to avoid the overspeed situation. In the simulation, $\ell$ is chosen as $\ell=0.5$. The value of the learning gain vector $\mathbf{L}$ is the same as that chosen in the pure D-type ILC (71).

In addition, to take input constraint into account simultaneously for the train operation simulation, the saturator $\operatorname{sat}(\cdot)$ in [41], which is defined as $\operatorname{sat}\left(u_{k}(t), u_{\text {max }}\right) \triangleq$ $\operatorname{sign}\left(u_{k}(t)\right) \min \left\{u_{\max },\left|u_{k}(t)\right|\right\}$, is added to the input force.

(4) Feedback control: The following feedback controller is also simulated, which has been typically and widely applied in practical train operation systems:

$$
u_{k}(t)=K_{p} e_{1, k}(t)+K_{d} \dot{e}_{1, k}(t)
$$

where the feedback gains tuned via error-and-try method are $K_{p}=0.3$ and $K_{d}=0.05$.

The simulation results of these four control schemes are shown as follows:

Figs. 6 and 7 demonstrate the tracking performance of these four schemes. The horizontal axis is the iteration number, and the vertical axis is the root mean square (RMS) of the tracking errors over the whole time interval. As seen from Figs. 6 and 7, the proposed AILC achieves better performance than the other three control schemes. The unknown system uncertainties make the pure feedback control inadequate to achieve satisfactory control performance. In addition, since the feedback control methods has no learning mechanism, it is hard to obtain an improved tracking precision no matter how many times the train repeats. In the D-type ILC and the D-type ILC with overspeed protection, the learning gains are fixed such that it is not adaptive to the system uncertainties. By comparison, the proposed AILC can cope with the uncertainties adaptively, and the projection mechanism used in the scheme prevents the unknown parameters being recklessly estimated, therefore an improved control performance can be achieved.

Figs. 8-11 show the speed tracking profiles of the four control schemes at the 10th and 30th iterations along with the desired speed trajectory. It is seen that the operation speed of the train by applying the pure D-type ILC and the pure feedback control exceeded the speed constraint in the 10th and 30th iterations, which violates the safe requirement of the train operation system. On the contrary, by using the proposed 


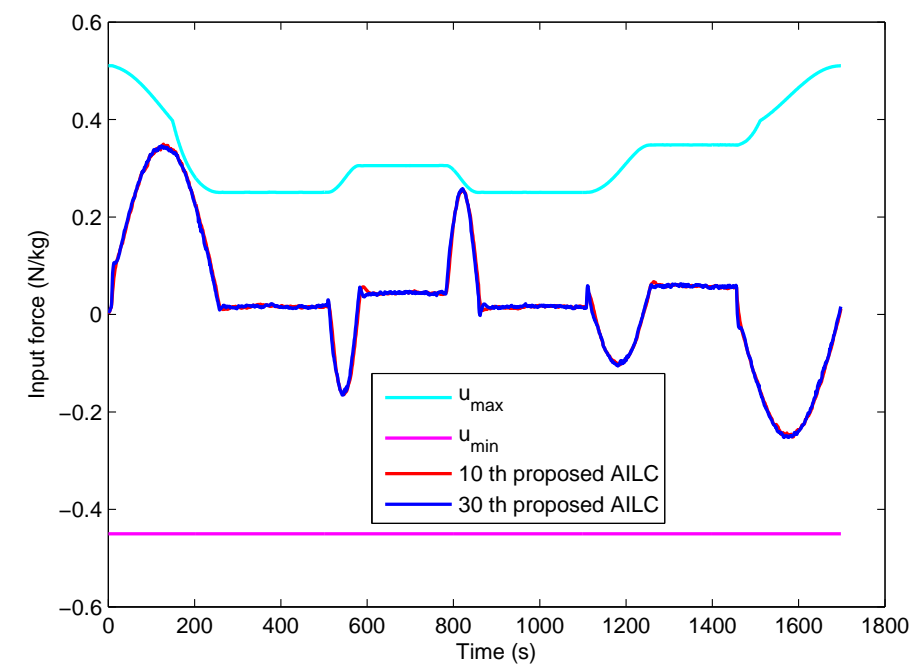

Figure 12: Control input profile of the proposed AILC at the 10th and 30th iterations.

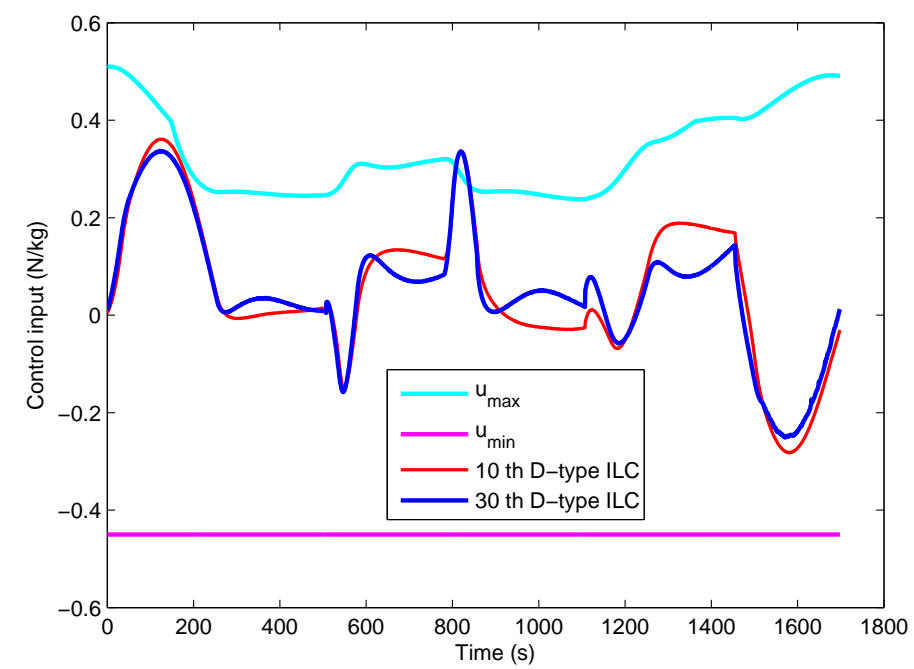

Figure 13: Control input profile of the D-type ILC at the 10th and 30th iterations.

AILC and the D-type ILC with overspeed protection, the operation speed can always abide by the speed constraint such that safe operation of the system can be guaranteed.

Figs. 12-15 give the required control input profiles of the four schemes at the 10th and 30th iterations. From Figs. 12-15, the input force generated by the pure D-type ILC and the pure feedback control exceeded the maximum traction force constraint in these 


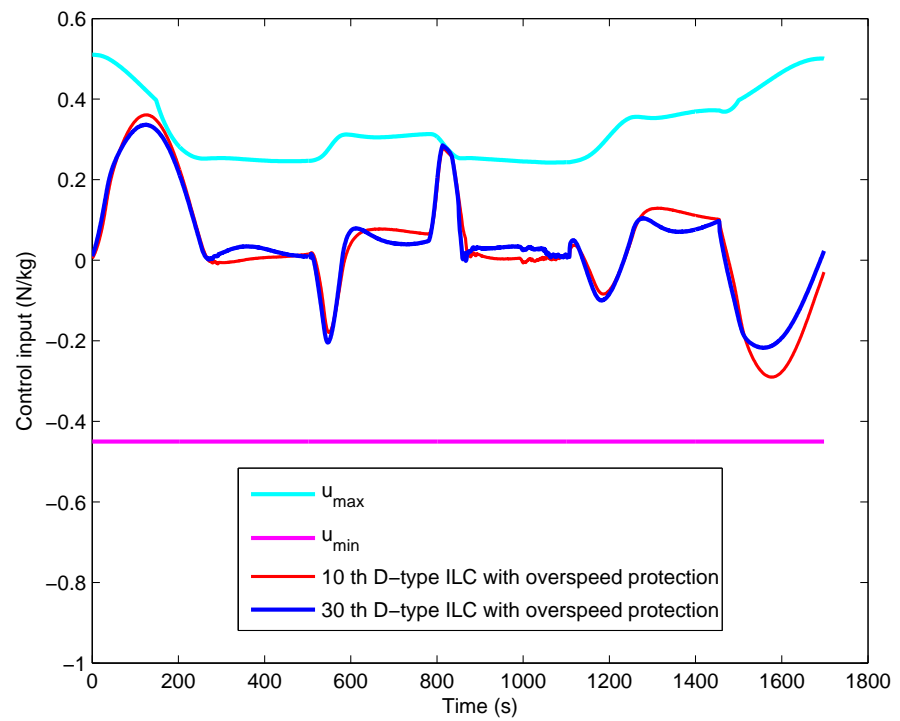

Figure 14: Control input profile of the D-type ILC with overspeed protection at the 10th and 30th iterations.

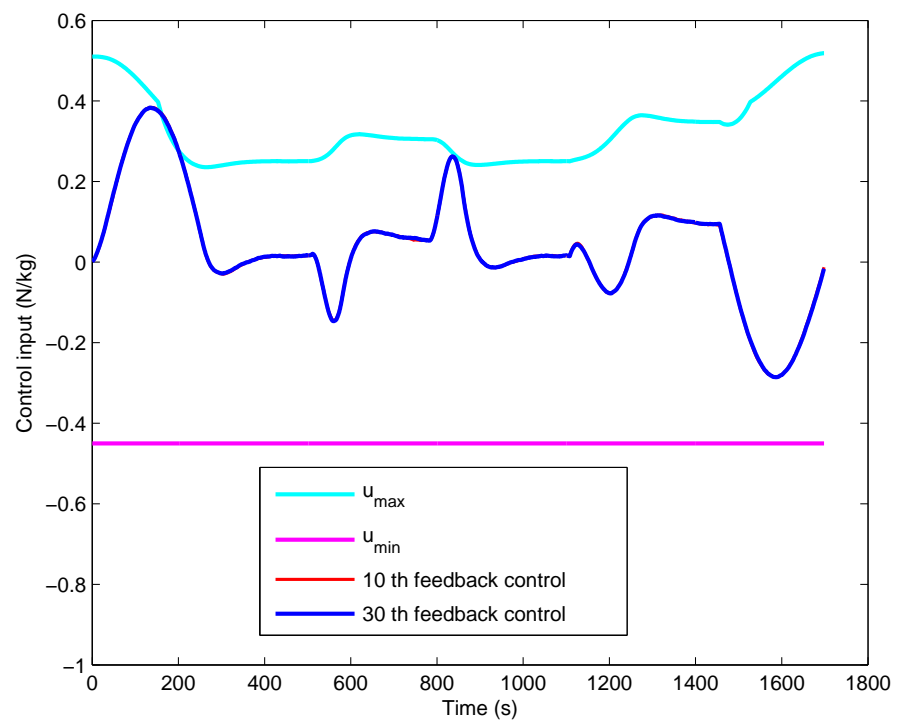

Figure 15: Control input profile of the feedback control at the 10th and 30th iterations.

two iterations. The input force generated by the D-type ILC with overspeed protection 
is constrained passively by the added saturator $\operatorname{sat}(\cdot)$. By contrast, the proposed AILC actively regulates the input force complying with the input constraints for any time in all iterations.

\section{Conclusion}

In this work, a novel AILC scheme is proposed for the tracking control of nonlinear systems with constraints and various uncertainties. The proposed AILC can actively restrict both the state and input to the given constrained ranges for all times and iterations. Meanwhile, the proposed method can guarantee the asymptotic convergence of the states to their desired ones theoretically, despite the presence of time-varying parametric uncertainties, unknown external disturbances and random initial state errors. Extensive simulation results of a train operation system are given to further verify the effectiveness of the proposed AILC. Some future issues in nonlinear AILC might be the topic on iteration-and-time-varying parametric uncertainties.

\section{Acknowledgements}

This work was supported by the National Science Foundation of China ( 61433002 , 61120106009, 61374102).

\section{References}

[1] C.J. Chien, A. Tayebi, Further results on adaptive iterative learning control of robot manipulators, Automatica 44 (3) (2008) 830-837.

[2] M. Butcher, A. Karimi, Linear parameter-varying iterative learning control with application to a linear motor system, IEEE/ASME Trans. Mechatronics 15 (3) (2010) 412-420.

[3] Z.S. Hou, J.W. Yan, J.X. Xu, Z.J. Li, Modified iterative-learning-control-based ramp metering strategies for freeway traffic control with iteration-dependent factors, IEEE Trans. Intell. Transp. Syst. 13 (2) (2012) 606-618.

[4] Y.Q. Wang, D.H. Zhou, F.R. Gao, Iterative learning model predictive control for multi-phase batch processes, J. Process Control 18 (6) (2008) 543-557.

[5] Z.S. Hou, Y. Wang, C.K. Yin, T. Tang, Terminal iterative learning control based station stop control of a train, Int. J. Control 84 (7) (2011) 1263-1274.

[6] H.Q. Sun, Z.S. Hou, D.Y. Li, Coordinated iterative learning control schemes for train trajectory tracking with overspeed protection, IEEE Trans. Autom. Sci. Eng. 10 (2) (2013) 323-333.

[7] Y.Q. Fan, W.Q. Wang, Y. Liu, Synchronization for a class of chaotic systems based on adaptive control design of input-to-state stability, International Journal of Innovative Computing, Information and Control 11(3) (2015) 803-814. 
[8] S. Oucheriah, Robust nonlinear adaptive control of a DC-DC boost converter with uncertain parameters, International Journal of Innovative Computing, Information and Control 11(3) (2015) 893-902.

[9] X.J Su, P. Shi, L.G. Wu, Y.D. Song, Fault Detection Filtering for Nonlinear Switched Stochastic Systems, IEEE Trans. Autom. Control, online available, DOI:10.1109/TAC.2015.2465091.

[10] M. Liu, Daniel W.C. Ho, P. Shi, Adaptive fault-tolerant compensation control for Markovian jump systems with mismatched external disturbance, Automatica 58 (2015) 5-14.

[11] Y. Wang, F. Gao, F.J. Doyle, Survey on iterative learning control, repetitive control, and run-to-run control, J. Process Control 19(10) (2009) 1589-1600.

[12] J.X. Xu, Z.S. Hou, On learning control: the state of the art and perspective, Acta Automatica Sinica 31 (6) (2005) 943-955.

[13] J. Hu, M. Tomizuka, Adaptive asymptotic tracking of repetitive signals-a frequency domain approach, IEEE Trans. Autom. Control 38 (10) (1993) 1572-1579.

[14] C. Kempf, W. Messner, M. Tomizuka, R. Horowitz, Comparison of four discretetime repetitive control algorithms, IEEE Control Syst. Mag. 13(6), (1993) 48-54.

[15] J. Y. Yao, Z. X. Jiao, D. W. Ma, A practical nonlinear adaptive control of hydraulic servomechanisms with periodic-like disturbances, IEEE/ASME Trans. Mechatronics, online available, DOI: 10.1109/TMECH.2015.2409893.

[16] J. Y. Yao, Z. X. Jiao, D. W. Ma, L. Yan, High-accuracy tracking control of hydraulic rotary actuators with modeling uncertainties, IEEE/ASME Trans. Mechatronics 19 (2) (2014) 633-641.

[17] J. Y. Yao, Z. X. Jiao, D. W. Ma, Adaptive robust control of DC motors with extended state observer, IEEE Trans. Ind. Electron. 61 (7) (2014) 3630-3637.

[18] S. Arimoto, S. Kawamura, F. Miyazaki, Bettering operation of robots by learning, J. Robot. Syst. 1 (2) (1984) 123-140.

[19] R.H. Chi, Z.S. Hou, J.X. Xu, Adaptive ILC for a class of discrete-time systems with iteration-varying trajectory and random initial condition, Automatica 44 (8) (2008) 2207-2213.

[20] M.X. Sun, D.W. Wang, Y.Y. Wang, Varying-order iterative learning control against perturbed initial conditions, J. Franklin Inst. 347 (8) (2010) 1526-1549.

[21] C.K. Yin, J.X. Xu, Z.S. Hou, A high-order internal model based iterative learning control scheme for nonlinear systems with time-iteration-varying parameters, IEEE Trans. Autom. Control 55 (11) (2010) 2665-2670. 
[22] X. Ruan, Z.Z. Bien, Q. Wang, Convergence characteristics of proportional-type iterative learning control in the sense of lebesgue-p norm, IET. Control Theory Appl. 6 (5) (2012) 707-714.

[23] D.Y. Meng, Y.M. Jia, J.P. Du, Coordination learning control for groups of mobile agents, J. Franklin Inst. 350 (8) (2013) 2183-2211.

[24] F.M. Chen, J.S.H Tsai, Y.T. Liao, S.M. Guo, M.C. Ho, F.Z. Shaw, L.S. Shieh, An improvement on the transient response of tracking for the sampled-data system based on an improved PD-type iterative learning control, J. Franklin Inst. 351(2) (2014) 1130-1150.

[25] F. Chen, R. Hou, G. Tao, Adaptive controller design for faulty UAVs via quantum information technology, Int. J. Adv. Rob. Syst. 9 (256) (2012) 1-7.

[26] F. Chen, B. Jiang, G. Tao, An intelligent self-repairing control for nonlinear MIMO systems via adaptive sliding mode control technology, J. Franklin Inst. 351 (1) (2014) 399-411.

[27] N. Amann, D.H. Owens, E. Rogers, Predictive optimal iterative learning control, Int. J. Control 69 (2) (1998) 203-226.

[28] J. Hätönen, D.H. Owens, Convex modifications to an iterative learning control law, Automatica 40 (7) (2004) 1213-1220.

[29] K.S. Lee, I.S. Chin, H.J. Lee, J.H. Lee, Model predictive control technique combined with iterative learning for batch processes, AIChE Journal 45 (10) (1999) 2175-2187.

[30] L.P. Wang, C.T. Freeman, S. Chai, E. Rogers, Predictive-repetitive control with constraints: From design to implementation, J. Process Control 23 (7) (2013) 956967.

[31] T.D. Son, H.S. Ahn, K.L. Moore, Iterative learning control in optimal tracking problems with specified data points, Automatica 49 (5) (2013) 1465-1472.

[32] C.T. Freeman, Y. Tan, Iterative learning control with mixed constraints for pointto-point tracking, IEEE Trans. Control Syst. Technol. 21 (3) (2013) 604-616.

[33] P. Janssens, G. Pipeleers, J. Swevers, A data-driven constrained norm-optimal iterative learning control framework for LTI systems, IEEE Trans. Control Syst. Technol. 21 (2) (2013) 546-551.

[34] A. Tayebi, C.J. Chien, A unified adaptive iterative learning control framework for uncertain nonlinear systems, IEEE Trans. Autom. Control 52 (10) (2007) 19071913.

[35] M.X. Sun, S.S. Ge, Adaptive repetitive control for a class of nonlinearly parametrized systems, IEEE Trans. Autom. Control 51 (10) (2006) 1684-1688. 
[36] X.-D. Li, T.-F. Xiao, H.-X. Zheng, Adaptive discrete-time iterative learning control for non-linear multiple input multiple output systems with iteration varying initial error and reference trajectory, IET. Control Theory Appl. 5 (9) (2011) 11311139.

[37] A. Tayebi, Adaptive iterative learning control for robot manipulators, Automatica 40 (7) (2004) 1195-1203.

[38] C. K. Yin, J.-X. Xu, Z.S. Hou, An ILC scheme for a class of nonlinear continuoustime systems with time-iteration-varying parameters subject to second-order internal model, Asian J. Control 13 (1) (2011) 126-135.

[39] C.J. Chien, A combined adaptive law for fuzzy iterative learning control of nonlinear systems with varying control tasks, IEEE Trans. Fuzzy Syst. 16 (1) (2008) $40-51$.

[40] J.X. Xu, X. Jin, State-constrained iterative learning control for a class of MIMO systems, IEEE Trans. Autom. Control 58 (5) (2013) 1322-1327.

[41] Y. Tan, J. Xu, M. Norrlöf, C. Freeman, On reference governor in iterative learning control for dynamic systems with input saturation, Automatica 47 (11) (2011) 2412-2419.

[42] R. Hunger, Floating point operations in matrix-vector calculus, Inst. for Circuit Theory and Signal Processing, Munich, Germany: Munich University of Technology, 2005.

[43] J.-J. E. Slotine, W.P. Li, Applied nonlinear control, Prentice-Hall, New York, 1991.

[44] K. B. Ngo, R. Mahony, Z.-P. Jiang, Integrator backstepping using barrier functions for systems with multiple state constraints, in Proc. 44th IEEE Conf. Decision Control, \& Eur. Control Conf. (2005) 8306-8312.

[45] K. P. Tee, S. S. Ge, E. H. Tay, Adaptive control of electrostatic microactuators with bidirectional drive, IEEE Trans. Contr. Syst. Technol. 17 (2) (2009) 340-352.

[46] K.P. Tee, S.S. Ge, E.H. Tay, Barrier lyapunov functions for the control of outputconstrained nonlinear systems, Automatica 45 (4) (2009) 918-927.

[47] W.J. Davis, Traction resistance of electric locomotives and cars, General Electric Rev. 29 (10) (1926) 685-708.

[48] W. W. Hay, Railroad engineering, Wiley, New York, 1982.

[49] H.R. Dong, B. Ning, B.G. Cai, Z.S. Hou, Automatic train control system development and simulation for high-speed railways, IEEE Circuits Syst. Mag. 10 (2) (2010) 6-18.

[50] Y. Chen, C. Wen, Z. Gong, M. Sun, An iterative learning controller with initial state learning, IEEE Trans. Autom. Control 44 (2) (1999) 371-376. 